\title{
A maternal-effect genetic incompatibility in Caenorhabditis elegans
}

\author{
Eyal Ben-David ${ }^{*} \dagger$, Alejandro Burga ${ }^{*}$, , Leonid Kruglyak ${ }^{\dagger}$
}

Department of Human Genetics, Department of Biological Chemistry, and Howard Hughes Medical Institute, University of California, Los Angeles, Los Angeles, CA 90095, USA.

†To whom correspondence should be addressed.Email: ebd@ucla.edu (E.B); aburga@mednet.ucla.edu (A.B.); 1kruglyak@mednet.ucla.edu (L.K.).

* These authors contributed equally to this work.

Selfish genetic elements spread in natural populations and have an important role in genome evolution. We discovered a selfish element causing a genetic incompatibility between strains of the nematode Caenorhabditis elegans. The element is made up of sup-35, a maternal-effect toxin that kills developing embryos, and pha-1, its zygotically expressed antidote. pha-1 has long been considered essential for pharynx development based on its mutant phenotype, but this phenotype in fact arises from a loss of suppression of sup-35 toxicity. Inactive copies of the sup-35/pha- 1 element show high sequence divergence from active copies, and phylogenetic reconstruction suggests that they represent ancestral stages in the evolution of the element. Our results suggest that other essential genes identified by genetic screens may turn out to be components of selfish elements. 


\section{Introduction}

Selfish genetic elements subvert the laws of Mendelian segregation to promote their own transmission (Dawkins 1976; Doolittle and Sapienza 1980; Orgel and Crick 1980; Werren 2011; Sinkins 2011). In what is perhaps the most extreme scenario, selfish elements can kill individuals that do not inherit them, leading to a genetic incompatibility between carriers and non-carriers (Beeman et al. 1992; Werren 1997, 2011; Hurst and Werren 2001; Lorenzen et al. 2008). Selfish elements are predicted to spread in natural populations (Hurst and Werren 2001; Werren 2011), and consequently, there is significant interest in using synthetic forms of such elements to drive population replacement of pathogen vectors in the wild (Chen et al. 2007; Hammond et al. 2015). However, despite the prominent role of genetic incompatibilities in genome evolution and their promise in pathogen control, their underlying genetic mechanisms have been resolved in only a few cases (Werren 2011). Our laboratory previously identified the only known genetic incompatibility in the nematode Caenorhabditis elegans (Seidel et al. 2008, 2011). The incompatibility is caused by a selfish element composed of two tightly linked genes: peel-1, a sperm-delivered toxin, and zeel-1, a zygotically expressed antidote. In crosses between isolates that carry the element and ones that do not, the peel-1 toxin is delivered by the sperm to all progeny, so that only embryos that inherit the element and the zeel-1 antidote survive. An analogous element, Maternal-effect dominant embryonic arrest (Medea) has been previously described in the beetle Tribolium; however, the underlying genes remain unknown (Beeman et al. 1992; Lorenzen et al. 2008).

\section{Results}

\section{A maternal-effect genetic incompatibility in $C$. elegans}

As part of ongoing efforts to study natural genetic variation in C. elegans, we introgressed a genetic marker located on the right arm of Chr. V from the standard laboratory strain N2 into the strain DL238 by performing eight rounds of backcrossing and selection. DL238 is a wild strain isolated in the Manuka Natural Reserve, Hawaii, USA, and is one of the most highly divergent C. elegans isolates identified to 
date (Andersen et al. 2012). To confirm the success of the introgression, we genotyped the resulting strain at single-nucleotide variants (SNVs) between DL238 and N2 by whole-genome sequencing. As expected, with the exception of a small region on the right arm of Chr. V where the marker is located, most of the genome was homozygous for the DL238 alleles (Fig. 1A). However, to our surprise, we observed sequence reads supporting the N2 allele at many SNVs on Chr. III, including two large regions that were homozygous for the N2 allele despite the eight rounds of backcrossing (Fig. 1A, Fig. S1). This observation suggested that N2 variants located on this chromosome were strongly selected during the backcrossing.

To investigate the nature of the selection, we performed a series of crosses between the N2 and DL238 strains and examined their progeny. To avoid effects of the peel-1/zeel-1 element, which is present in N2 and absent in DL238, we performed a cross between DL238 males and a near isogenic line (NIL) that lacks the peel-1/zeel-1 element in an otherwise N2 background (hereafter, N2 peel-1 ${ }^{-/}$) (Seidel et al. 2011). We observed low baseline embryonic lethality in the $\mathrm{F}_{1}$ generation and in the parental strains $(0.26 \%(N=381)$ for $\mathrm{F}_{1} ; 0.99 \%(N=304)$ for DL238; $0.4 \%(N=242)$ for $\mathrm{N} 2$ peel- $\left.-^{-/}\right)$, and we did not observe any obvious abnormal phenotypes in the $F_{1}$ that could explain the strong selection. However, when we allowed heterozygous $\mathrm{F}_{1}$ hermaphrodites from this cross to self-fertilize, we observed $25.15 \%(N=855)$ embryonic lethality among the $\mathrm{F}_{2}$ progeny (Fig. 1B). Similar results were obtained for F1 hermaphrodites from the reciprocal parental cross $(26.1 \%, N=398)$. These results suggested the presence of a novel genetic incompatibility between N2 and DL238 that causes embryonic lethality in their $\mathrm{F}_{2}$ progeny.

The observed pattern of embryonic lethality (no lethality in the parents nor in the $\mathrm{F}_{1} ; 25 \%$ lethality in the $F_{2}$ ) is consistent with an interaction between the genotype of the zygote and a maternal or paternal effect (Fig. 1C) (Seidel et al. 2008). We hypothesized that the incompatibility could stem from a cytoplasmicallyinherited toxin that kills embryos if they lack a zygotically expressed antidote, analogous to the mechanism of the peel-1/zeel-1 element (Seidel et al. 2008, 2011). To test this model and to discriminate between maternal and paternal effects, we crossed heterozygous $\mathrm{F}_{1}$ DL238 x N2 peel-1 $1^{-/}$males and hermaphrodites 
with DL238 hermaphrodites or males, respectively (Fig. 1B, Fig. S2). We observed $48.59 \%(N=389)$ lethality when $\mathrm{F}_{1}$ hermaphrodites were crossed to DL238 males, but only baseline lethality $(1.17 \% ; N=$ 171 ) in the reciprocal cross of $F_{1}$ males to DL238 hermaphrodites. $50 \%$ lethality when the $F_{1}$ parent is the mother and no lethality when the F1 parent is the father indicates that the incompatibility is caused by maternal-effect toxicity that is rescued by a linked zygotic antidote (Fig. S2). We tested whether the new incompatibility was independent from the paternal-effect peel-1/zeel-1 element by crossing DL238 and N2 worms and selfing the $\mathrm{F}_{1}$ progeny. We observed $41.37 \%(N=389)$ embryonic lethality among the $\mathrm{F}_{2}$ progeny, consistent with expectation for Mendelian segregation of two independent incompatibilities (43.75\%) (Fig. 1D).

\section{pha-1 and sup-35 constitute a selfish element that underlies the incompatibility between DL238 and}

\section{N2}

To identify the genes underlying the maternal-effect incompatibility between N2 and DL238, we sequenced the genome of DL238 using Illumina short reads and aligned those reads to the N2 reference genome. We focused our attention on the two regions on Chr. III that were completely homozygous for the N2 allele in the introgressed strain (Fig. 1A, Fig. S1). Inspection of short read coverage revealed a large $\sim 50 \mathrm{~kb}$ region on the right arm of the chromosome with very poor and sparse alignment to the N2 reference (Chr III: 11,086,500 - 11,145,000) (Fig. 2A). This region contains ten genes and two pseudogenes in N2. We noticed that pha-1, annotated as an essential gene in the reference genome, appeared to be completely missing in DL238 (Fig. 2A) (Schnabel and Schnabel 1990). pha-1 was originally identified as an essential gene required for differentiation and morphogenesis of the pharynx, the C. elegans feeding organ (Schnabel and Schnabel 1990). But if pha-1 is essential for embryonic development and missing in DL238, then how are DL238 worms alive? pha-1 lethality can be fully suppressed by mutations in three other genes: sup-35, sup36, and sup-37 (Schnabel et al. 1991). We found no coding variants in sup-36 and sup-37 which reside on 
chromosomes IV and V, respectively (Schnabel et al. 1991) (Fig. S3). However, sup-35, which is located 12.5kb upstream of pha-1, also appeared to be missing or highly divergent in DL238 (Fig. 2A, Fig. S3).

We hypothesized that sup-35 and pha-1 could constitute a selfish element responsible for the observed incompatibility between the N2 and DL238 isolates. In our model, sup-35 encodes a maternally-deposited toxin that kills embryos unless they express the zygotic antidote, pha-1 (Fig. 2B). N2 worms carry the sup35/pha-1 element, which is missing or inactive in DL238, and $\mathrm{F}_{1}$ hermaphrodites deposit the sup-35 toxin in all their oocytes. $25 \%$ of their $F_{2}$ self-progeny do not inherit the element and are killed because they lack the antidote pha-1. Consistent with our model, an RNA-sequencing time-course of C. elegans embryogenesis showed that sup-35 transcripts are maternally provided, whereas pha-1 transcripts are first detected in the embryo at the 100-cell stage (Hashimshony et al. 2014). To test our model, we first asked whether sup-35 was necessary for the $\mathrm{F}_{2}$ embryonic lethality in the N2 x DL238 cross. We crossed DL238 males to N2 peel-1/- hermaphrodites carrying a null sup-35(e2223) allele (Fig. 2B). This sup-35 allele was reported to fully rescue pha-1 associated embryonic lethality (Schnabel et al. 1991). Embryonic lethality in the $\mathrm{F}_{2}$ dropped from $25 \%$ to baseline in this cross $(1.40 \%, N=576)$, demonstrating that sup-35 activity underlies the incompatibility between N2 and DL238 (Fig. 2B). We next tested whether expression of pha1, the zygotic antidote, was sufficient to rescue the embryonic lethality. We introgressed a pha-1 multicopy transgene into the DL238 and N2 peel-1/- $1^{-/}$strains and repeated the cross. As predicted, expression of pha-1 was sufficient to reduce embryonic lethality in the $\mathrm{F}_{2}$ to baseline $(1.49 \%, N=268)$ (Fig. 2B). Moreover, we reasoned that if the sup-35/pha-1 element underlies the maternal incompatibility, arrested embryos from an N2 x DL238 cross should phenocopy pha-1 mutant embryos. We collected rare L1-arrested $\mathrm{F}_{2}$ larvae from an N2 peel-1 $1^{-/}$x DL238 cross and observed major morphological defects in the pharynx of these individuals, as previously reported for pha-1 mutants (Schnabel and Schnabel 1990; Polley et al. 2014) (Fig. 2C). 
Together, these results show that sup-35 toxicity underlies the incompatibility between N2 and DL238, and that sup-35 and pha-1 constitute a selfish element in C. elegans. Moreover, our results indicate that pha-1 is not an organ-specific differentiation gene, as originally proposed (Schnabel and Schnabel 1990), but a zygotically expressed antidote, and sup-35 is a maternal-effect toxin rather than a suppressor of pha-1. This major reinterpretation of the roles of pha-1 and sup-35 is strongly supported by multiple lines of evidence from previous studies (Mani and Fay 2009; Fay et al. 2012; Kuzmanov et al. 2014; Polley et al. 2014). First, sup-35 overexpression phenocopies pha-1 mutations, showing that sup-35 is sufficient to cause embryonic lethality (Mani and Fay 2009). Second, all defects associated with pha-l mutations are suppressed by mutations in sup-35 (Mani and Fay 2009; Fay et al. 2012; Kuzmanov et al. 2014; Polley et al. 2014). Third, when $\mathrm{N} 2$ hermaphrodites heterozygous for a deletion that includes both sup-35 and pha$1(t D f 2 /+)$ self, the $25 \%$ of their progeny that are homozygous for this deletion arrest as embryos with pharyngeal defects (Schnabel et al. 1991; Mani and Fay 2009). Importantly, the lethality and pharyngeal defects of those homozygous embryos can be rescued by growing the heterozygous $t D f 2 /+$ mother in sup35 RNAi, which depletes sup-35 transcripts from the germline (Fay et al. 2012). These results indicate that maternally deposited sup-35 is sufficient to kill embryos that lack pha-1, which is consistent with the role of sup-35 as a maternal-effect toxin. Finally, pha-1 is not found in any other nematode sequenced to date (Fig 2A). This observation is more consistent with its recent evolution as part of a selfish element in $C$. elegans than with its previously postulated role as a key developmental regulator (Schnabel and Schnabel 1990).

\section{Global variation in the activity of the sup-35/pha-1 element}

We examined the sequences of sup-35 and pha-1 in 152 C. elegans wild isolates that represented unique isotypes (Cook et al. 2016) in the Caenorhabditis elegans Natural Diversity Resource (Cook et al. 2017).

Two isolates, QX1211 (California, USA) and ECA36 (Auckland, New Zealand), harbored a highly mutated copy of pha-1, with multiple non-synonymous SNVs as well as frameshifts expected to completely disrupt 
the protein (Fig. S3 and S4). Both of these isolates also appeared to be missing sup-35 (Fig. S3). We predicted that these isolates should be incompatible with N2. Because QX1211 and ECA36 carry the same haplotype in the sup-35/pha-1 region, we focused on further characterizing QX1211. We crossed QX1211 to $\mathrm{N} 2$ peel- $1^{-/}$worms and observed $23.9 \%(N=355)$ lethality in the $\mathrm{F}_{2}$ progeny, consistent with QX1211 carrying a degenerate copy of pha-1. Importantly, the lethality was abolished when we crossed QX1211 with N2 sup-35(e2223); peel-1 $1^{--}(0 \%, N=290)$. Furthermore, we observed background levels of embryonic lethality $(1.0 \%, N=294)$ in the $\mathrm{F}_{2}$ progeny of a DL238 x QX1211 cross, as expected, since both strains lack functional sup-35. Our analysis also revealed that the highly divergent Hawaiian isolate CB4856 carried a functional sup-35/pha-1 element, which explains why previous studies did not detect this incompatibility (Seidel et al. 2008). Consistent with this observation, crossing the CB4856 and DL238 isolates led to the expected embryonic lethality in the $\mathrm{F}_{2}(22.3 \%, N=349)$.

We looked for additional variation in sup-35, sup-36, and sup-37 across the 152 isolates, which could potentially affect the activity of the sup-35/pha-1 element (Fig. S5-7). We found eight non-synonymous variants (three in sup-35, three in sup-36 and two in sup-37) and one premature stop codon in sup-35 that removed $47 \%$ of the protein. We also identified potential deletions by visually inspecting read alignments in each of the 152 isolates. While sup-36 and sup-37 had consistent coverage in all isolates, we identified two structural variants in sup-35: a $530 \mathrm{bp}$ deletion in the third intron, and a large $12.1 \mathrm{~kb}$ deletion that removed part of the last exon and the 3' UTR and fused sup-35 to Y48A6C.1, a pseudogene that has partial homology with sup-35, creating a chimeric transcript (Fig. S8 and S9). We tested strains carrying each of these variants for a maternal-effect incompatibility with DL238 (Table S2, Fig. S10), and found that the incompatibility was completely abolished in strains carrying the chimeric sup-35/Y48A6C.1 gene and in the strain carrying the premature stop codon in sup-35, indicating that these variants disrupt sup-35 function. Thus loss of sup-35 activity has occurred independently at least twice in carriers of N2-like alleles the element. 


\section{DL238 and QX1211 carry an ancestral sup-35/pha-1 haplotype}

The alignment of DL238 and QX1211 short reads to the N2 reference genome was very sparse throughout the sup-35/pha-1 region and at nearby genes, with some genes aligning only in exons and others not aligning at all (Fig. 2A). Moreover, several attempts to define the boundaries of the pha- 1 deletion in DL238 using diverse combinations of PCR primer pairs were unsuccessful. This suggested that the DL238 and QX1211 haplotypes were highly divergent from the N2 reference, and that major genomic rearrangements may have occurred. To resolve the genomic structure of the sup-35/pha-1 element in these isolates, we de novo assembled the genomes of DL238 and QX1211 using a combination of our and previously published Illumina short reads (van Schendel et al. 2015; Cook et al. 2017), followed by targeted Sanger sequencing to resolve repetitive regions and confirm scaffolds. The de novo assemblies confirmed that pha- 1 is absent from DL238 and is highly pseudogenized in QX1211, and that sup-35 is pseudogenized in both (Fig. 3, Fig. S11). DL238 and QX1211 share a very similar haplotype, with the exception of a large deletion in DL238 that encompasses pha-1, fbxa-128 and several exons of Y47D3A.1 (Fig. S11). We also identified other large structural variants in both DL238 and QX1211 at the sup-35/pha-1 locus. First, relative to the $\mathrm{N} 2$ reference genome, nearly $20 \mathrm{~kb}$ of sequence is missing completely from both isolates (Fig. S11). Second, the region spanning the pseudogenized sup-35 and Y48A6C.4 is inverted relative to the N2 reference (Fig. 3, Fig. S11). This inversion was confirmed using single molecule Oxford Nanopore long-read sequencing (Fig. S12). As a consequence of the inversion, the pseudogenized sup-35 and pha-1 are located next to each other in QX1211, rather than flanking Y48A6C.4 as in the N2 reference genome (Fig. 3, Fig. S11).

To gain further insights into evolution of the sup-35/pha-1 element, we aligned the N2, DL238 and QX1211 haplotypes to the homologous regions of diverse Caenorhabditis species, using the highly conserved genes (hmt-1, Y48A6C.4, and Y47D3A.29) that delineated the region (Fig. 3, Fig. S11). Unexpectedly, our analysis revealed that the order and orientation of these three genes in the other Caenorhabditis species matched that in DL238 and QX1211 rather than the order and orientation in N2. This observation suggests that the 
sup-35/pha-1 haplotype in DL238 and QX1211 derives from an early stage in the evolution of the selfish element, which was followed by a major inversion that now defines the N2 haplotype, and subsequent degeneration of the element in DL238 and QX1211. In further support of this model, a gene tree built using the coding region of Y48A6C.4 from all the C. elegans isolates and the other Caenorhabditis species showed that DL238, QX1211 and ECA36 cluster in a separate branch from all other C. elegans isolates (Fig. 3).

\section{Discussion}

We discovered a genetic incompatibility in C. elegans that is caused by an interaction between a maternally deposited toxin, sup-35, and a zygotically expressed antidote, pha-1. The antidote, pha-1, was originally thought to be a developmental gene, in large part due to the specific pharyngeal defects observed in mutants (Schnabel and Schnabel 1990; Granato et al. 1994; Okkema et al. 1997; Fay et al. 2004; Mani and Fay 2009). However, the precise role of pha-1 in embryonic development remained elusive and controversial (Mango 2009; Fay et al. 2012; Kuzmanov et al. 2014). Our results indicate that pha-1 pharyngeal defects are a direct consequence of sup-35 toxicity, and that sup-35 and pha-1 act as a selfish element, instead of being integral components of C. elegans embryonic development as originally suggested.

One important insight emanating from previous work in light of our results is that the sup-35/pha-1 element exerts its toxicity by recruiting genes that are directly involved in C. elegans development (Schnabel et al. 1991; Fay et al. 2004, 2012; Mani and Fay 2009; Polley et al. 2014). The other two known suppressors of pha-1 lethality, sup-36 and sup-37, are essential for sup-35 toxicity and are conserved in other nematodes (Fay et al. 2012; Polley et al. 2014). Interestingly, sup-37 is required for normal pharyngeal pumping and promotes ovulation in the somatic gonad independently of pha-1 function (Fay et al. 2012). Null sup-37 mutants are inviable and undergo early larval arrest. However, a single missense and viable mutation in sup-37 is sufficient to abolish sup-35 toxicity (Fay et al. 2012; Polley et al. 2014). Together with the finding that SUP-37 physically interacts with SUP-35 (Polley et al. 2014), this suggests that the sup-35/pha-1 
selfish element is hijacking a developmental pathway to kill those embryos that do not inherit it. The specificity in the activity and expression of sup-36 and sup-37 may explain the pharyngeal phenotypes of pha-1 mutants. We hypothesize that PHA-1 could act as an antidote by directly inhibiting the interaction between SUP-35 and SUP-37. The transcription factor lin-35/Rb and the E2 ubiquitin conjugation enzyme $u b c-18$ downregulate sup-35 (Mani and Fay 2009). An attractive possibility is that this regulation evolved as an additional mechanism to cope with sup-35 toxicity, as part of an arms race between the selfish element and its host. Future studies may further resolve the mechanism of sup-35 toxicity and its regulation.

One of the most intriguing aspects of toxin-antidote systems is their origin. The study of the pha-1/sup-35 element provides some clues. pha-1 has no homology to any other gene outside C. elegans. On the other hand, sup-35 is a homolog of another C. elegans gene, rmd-2, which is conserved in other nematodes (Mani and Fay 2009). A phylogenetic analysis shows that sup-35 is more closely related to C. elegans rmd-2 than to $r m d-2$ genes from other species, and is likely a paralog of $r m d-2$ (Fig. S13 and S14). These results suggest that the origin of the sup-35/pha-1 element involved the duplication of a pre-existing gene (rmd-2) and the recruitment of a novel gene of unknown origin in the lineage leading to C. elegans.

Among 152 C. elegans wild isolates examined, only DL238, QX1211, and ECA36 do not carry the derived inversion in the sup-35/pha-1 element, and in all three of them, the selfish element is highly pseudogenized. Similar inversions have been described in the Drosophila segregation distorter locus and in the mouse $t$ haplotypes (Shin et al. 1983; Hurst and Werren 2001; Larracuente and Presgraves 2012), and are thought to stabilize two-component driver systems by preventing recombination from decoupling the components (Hurst and Werren 2001). Has the inversion facilitated the spread of sup-35/pha-1 through the C. elegans population to all but few isolates? Ongoing efforts to identify more divergent isolates, as well as nematode species that are more closely related to C. elegans, may fill in the gaps in our understanding of the evolution of this element. 
Lastly, our work highlights the importance of studying natural genetic variation for understanding gene function. Despite the indisputable value of a common reference strain, it has proved extremely difficult in the context of the N2 background alone to either confirm or rule out pha-1 as an essential component of $C$. elegans embryonic development. The study of other wild isolates has made possible our characterization of sup-35/pha-1 as a selfish element. Our results show that some essential genes may, in fact, turn out to be antidotes to unknown toxins. Selfish elements conferring genetic incompatibilities may be more common than previously thought, and some of them may be hiding in plain sight.

\section{Acknowledgments:}

We thank members of the Kruglyak lab for their comments. Funding was provided by the Howard Hughes Medical Institute and NIH grant R01 HG004321 (L.K.). E.B. is supported by a Gruss-Lipper postdoctoral fellowship from the EGL foundation. A.B. is supported by the Jane Coffin Childs Memorial Fund for Medical Research. E.B., AB. and L.K. wrote the manuscript. All authors discussed and agreed on the final version of the manuscript. The authors declare no competing financial interests.

\section{Methods}

\section{C. elegans strains and mutant alleles}

Strains were grown using standard culturing techniques, with the exception that a modified nematode growth medium (NGM) containing $1 \%$ agar and $0.7 \%$ agarose was used to prevent burrowing of wild isolates (Brenner 1974; Andersen et al. 2015). All experiments were carried out at $20^{\circ} \mathrm{C}$. All the strains used and generated in this study are listed in Table S2. Some of the strains were provided by the CGC, which is funded by the NIH Office of Research Infrastructure Programs (P40 OD010440). The construction of strains carrying the peel-1/zeel-1 allele from CB4856 (niDf9) was performed by backcross following a PCR product specific to the N2 allele 
amplified using the following primers: FW GCAGAGGAGGCAAAGGTGACTA; RV AGCACGTGTAGGCAGAAGTCAT.

\section{Introgression of a Chr. V genetic marker into DL238}

We introgressed the fog-2 (q71) allele from the N2 background into DL238 by performing eight consecutive rounds of backcross and selection for the feminization of the germline (fog) phenotype (Schedl and Kimble 1988). Since the peel-1/zeel-1 element is active in N2 but not in DL238, we performed the backcross using DL238 males and feminized hermaphrodites. The use of DL238 males avoided the fixation of the peel-1/zeel-1 element on Chr. I because DL238 worms do not carry the PEEL-1 toxin in their sperm. Consequently, the fixation of the N2 haplotype on Chr. III in the introgression strain could not be caused a paternal-effect toxin. The introgressed marker, fog-2(q71), is required for spermatogenesis in hermaphrodites but not in males, and the resulting strain must reproduce by outcrossing. The observed pattern of linkage decay around the N2 homozygous region on Chr. III (fig. S1) is a consequence of selection acting in an obligate outcrossing population.

\section{Short read sequencing}

We extracted genomic DNA (gDNA) using the DNeasy Blood \& Tissue kit (Qiagen). We prepared Illumina sequencing libraries using the Nextera protocol (Illumina). We followed the standard protocol with the following exception: we performed agarose size-selection of the Nextera libraries, extracting a $\sim 500$ bp band. Libraries were quantified using the Qubit HS kit and sequenced using 300bp paired-end V3 kits on a Miseq desktop sequencer (Illumina) at 12 pM. In total, we generated 29,998,002 reads for DL238 and 14,428,754 reads for QX2323. 


\section{Variant calling in DL238}

Automated preprocessing, alignment and variant calling were done using bcbio-nextgen (ver. 0.9.9) (https://bcbio-nextgen.readthedocs.io/). Short reads from DL238 were aligned to the WBcel235 build of the reference N2 genome. Variant calling was performed with four different software packages: GATK HaplotypeCaller (McKenna et al. 2010), Platypus (Rimmer et al. 2014), Freebayes (Garrison and Marth 2012) and Varscan (Koboldt et al. 2012). Variants identified by fewer than three of the callers were filtered out, resulting in 239,131 SNVs between DL238 and $\mathrm{N} 2$.

\section{Genotyping of the DL238 isolate and the DL238 Chr. V introgression strain}

Analyses were performed using the R Project for Statistical Genetics (https://www.r-project.org/). Plotting was done using the R package ggplot2 (Wickham 2009) and genomic data was plotted using the R package gviz (Hahne and Ivanek 2016). We sought to accurately assess the success of the introgression genome-wide, rather than exhaustively identify variants. Therefore, we implemented a highly stringent filtering pipeline to extract only the highest confidence SNVs from our initial list of 239,131. First, we used all variants (SNVs and Indels) to generate an alternative DL238 reference build. We extracted a 50bp region around each SNV from the N2 reference genome and aligned it to the DL238 alternative reference using bowtie (ver. 1) (Langmead et al. 2009), allowing up to 5 mismatches. This was implemented using the R package liftove $R$ we have developed (https://github.com/eyalbenda/liftoveR), and allowed us to eliminate SNVs that were proximal to structural variants (which wouldn't align with bowtie). That reduced our set to 141,073 SNVs. We aligned both the QX2323 and the DL238 sequencing reads using burrows wheeler 
aligner (bwa) (Li and Durbin 2010) to the N2 and the DL238 references, and removed PCR duplicates using Picard. We then counted the number of reads supporting each allele of each of the 141,073 SNVs identified in DL238 using bam-readcount (https://github.com/genome/bamreadcount). To reduce the number of spurious SNVs, we restricted our analysis to SNVs for which all DL238 reads supported the DL238 allele when aligning to DL238, and no reads supported the N2 allele when aligning to N2. This reduced the number of SNVs to 53,186. Finally, to accurately assess the dosage of each allele, SNVs with less than 20x coverage were filtered out. 44,200 SNVs passed filtering. The fraction of reads with the N2 allele at these SNVs was plotted without smoothing.

\section{Mating and embryonic lethality scoring in the crosses between DL238 and N2 peel-1/-}

Males and L4 hermaphrodites were picked onto an NGM plate seeded with a small drop of OP50 bacteria and left to mate. We used a high male to hermaphrodite ratio (at least 5:1), and inspected the $F_{1}$ progeny to ensure both sexes were equally represented. To score embryonic lethality in the progeny of selfing $\mathrm{F}_{1}$ hermaphrodites, $\mathrm{F}_{1} \mathrm{~L} 4$ hermaphrodites were transferred to a new plate. Next morning, gravid hermaphrodites were allowed to lay eggs for 4-8 hours. Eggs laid during this time window were collected and manually transferred to a new unseeded plate, counted and scored 1820 hours later. Lethality was defined as the percentage of unhatched eggs. Embryonic lethality in the progeny of mating hermaphrodites was scored similarly, but L4 hermaphrodites were transferred together with males to a new plate, and their eggs were laid and collected in the presence of these males to guarantee continuous mating.

\section{Mapping of the genetic incompatibility}


To identify the locus underlying the strong selection in favor of the N2 haplotype on Chr. III, we first identified regions homozygous for the N2 alleles in the QX2323 introgression strain using our curated SNV list. We identified two regions: Chr III. 3,302,436-3,607,723 and Chr. III. 10,964,134-11,254,095 (Fig. S1). Of these two regions, only the one located toward the right arm of Chr. III showed a pattern of decaying linkage, suggesting that this region was more likely to harbor the causal locus. The homozygous N2 region on the left arm of Chr. III could be the result of an unknown structural variant in DL238 unrelated to the incompatibility. Visual inspection of DL238 short read alignments in the homozygous region revealed that pha- 1 and sup-35 were very likely missing in DL238. To test whether sup-35 was necessary for the genetic incompatibility, we used the GE346 strain carrying the sup-35(e2223) allele that was shown to suppress pha-1 mutations (Schnabel et al. 1991). The strain also carried in close linkage a temperature sensitive (ts) pha-1(e2123) allele, as well as a recessive $d p y-18(\mathrm{e} 499)$ mutation that caused a dumpy (dpy) phenotype. All crosses were performed at $20^{\circ}$, the permissive temperature of the pha-1(e2123) allele. $F_{1}$ cross progeny from $d p y$ hermaphrodites were confirmed by lack of the $d p y$ phenotype. Since GE346 carries the active N2 peel-1/zeel-1 allele, we crossed GE346 into the N2 peel-1/strain, selecting for $d p y$ and the absence of peel-1. Hermaphrodites of the resulting strain (QX2329) were crossed to DL238 males. To test whether transgenic expression of pha-1 was sufficient to rescue the embryonic lethality, we used the OH10050 strain carrying the integrated extrachromosomal array otIs317 $[m g l-1:: m$ Cherry + pha- $1(+)]$. We introgressed the transgene from $\mathrm{OH} 10050$ into DL238 by performing eight rounds of backcross and selection for $m$ Cherry fluorescence. We also introgressed the transgene into the N2 peel- $1^{-/-}$strain, and crossed the two strains. This guaranteed expression of the transgene in all $\mathrm{F}_{2}$ progeny. 


\section{Microscopy}

We selected phenotypically WT and rare L1 arrested embryos from the F2 of an N2 peel-1/- $\mathrm{x}$ DL238 cross. Larvae were transferred to a 3\% agarose pad and visualized under bright field using a Nikon Eclipse 90i microscope equipped with a Photometrics CoolSNAP HQ2 CCD camera.

\section{Screening for variants in sup-35, sup-36 and sup-37 across wild isolates}

Raw Illumina short reads sequencing data from 152 isolates (Cook et al. 2017) was acquired from the Sequence Read Archive (SRA) (Bioproject PRJNA318647). Alignment and variant calling were done using bcbio-nextgen as described above for DL238 to identify coding variants in sup35, sup-36 or sup-37. To identify structural variation, we visualized the alignment in each of these three genes across the isolates, finding two structural variants in sup-35 (fig. S5,S8 and S9). The variants were visualized using mutation "lollipop" plots generated using the trackViewer Bioconductor R package. Multiple sequence alignments were visualized using Geneious ver. 10 (restricted free version) (https://www.geneious.com/).

\section{Screening for sup-35/pha-1 activity in isolates carrying variants in sup-35, sup-36 or sup-37}

To facilitate screening wild isolates for variants that abolish the incompatibility with DL238, we used a fog-2(q71) introgression strain in the DL238 background (QX2327) that was homozygous for the DL238 sup-35/pha-1 allele. This strain was generated by backcrossing the QX2323 strain into DL238 for two additional generations using QX2323 males and DL238 hermaphrodites, thus avoiding the maternal incompatibility. The QX2327 strain was genotyped to ensure it was homozygous for the DL238 sup-35/pha-1 allele. This strain is obligate outcrossing, and we crossed QX2327 hermaphrodites to males from each of the wild isolates, thereby ensuring that the progeny 
in the next generation resulted exclusively from crossing and not selfing. We noted that the baseline lethality in this strain was markedly higher than wild-type $(4.7 \%, N=252)$. However, when we backcrossed QX2327 to DL238, and allowed the F1 to self, the lethality was reduced back to background levels $(0.5 \%, N=362)$. These results explain why higher lethality $(>50 \%)$ is observed in crosses in which $\mathrm{F}_{1}$ males are backcrossed to QX2327 hermaphrodites, while the expected lethality is observed in the selfing $F_{1}$ and in the reciprocal backcrosses that use $F_{1}$ hermaphrodites (Table S1).

In a screen for functional mutations in sup-35, we expect null sup-35 alleles to reduce the lethality from $25 \%$ to baseline levels in the $\mathrm{F}_{2}$. In contrast, even null variants in sup-36 or sup-37, which are unlinked to sup-35/pha-1, are predicted to only reduce the embryonic lethality from $25 \%$ to $18.75 \%$ if they are recessive. This is due to the fact that of the $\mathrm{F}_{2}$ progeny that do not inherit pha$1(25 \%), 75 \%$ will inherit at least one functioning copy of the sup-36 (or sup-37) allele. Furthermore, in a backcross of $F_{1}$ males to QX2327 hermaphrodites, all of the $F_{2}$ progeny inherit at least one functioning copy of sup-36 (or sup-37) from the QX2327 parent, and the lethality isn't expected to be reduced at all. For these reasons, and given the sample sizes in our screening, we cannot rule out the presence of hypomorphic variants weakly affecting sup-35, or even strongly affecting sup-36 and sup-37, in some of the wild isolates tested.

\section{Validation of chimeric fusion}

To validate the large deletion leading to the fusion of sup-35 and the Y48A6C.1 pseudogene, we designed primers that flanked the deletion: FW-del: GATCACGTGAGACAGGAAAAG and RVdel: CCCTTCAAAAGCACACCAAC. This primer pair amplified the expected $1000 \mathrm{bp}$ band in 
the wild isolate ED3012, which carries the deletion, but not in the reference N2 (fig. S8C). As a positive control, we amplified the pha-l locus using primers FW-pha-1: CCGTTTTCATCACGTTGCTCGA and RV-pha-1: TGTCGCGCACTACTGAATCAGA. To confirm whether the chimeric fusion sup-35/Y48A6C.1 is expressed, we performed reverse transcription (RT) PCR (fig. S8D). Total RNA was isolated from mixed stage N2 and ED3012 populations using the RNeasy kit (Qiagen), and cDNA was prepared using the SuperScript III Reverse Transcriptase kit (Thermo Fisher Scientific). We used primers FW1: TTTTTCGCTTTCCAAACTGG, RV1: GCGAGCAACTCTTTCTCGAT, RV2: ATTTTGAGAGCAAGCCGAAA. The FW1-RV1 primer pair amplifies exclusively the spliced cDNA of wild type sup-35, and the FW1-RV2 primer pair amplifies exclusively the spliced cDNA of the sup-35/Y48A6C.1 chimeric fusion. As a positive control, we amplified the pha-1 transcript using the primers FW-pha-1.2: CGGACCAGTTCAAAATGACA and RV-pha-1.2: TTTTCTGCTGGGAGATTTGC. The primers span multiple exons, allowing us to distinguish the spliced cDNA PCR product.

\section{De novo genome assembly}

We de novo assembled the DL238 and QX1211 genomes by combining Illumina short reads generated by two previous studies available at the Sequence Read Archive (Bioproject PRJNA318647 and PRJNA260487) (van Schendel et al. 2015; Cook et al. 2016). Average insert size in those libraries was $200 \mathrm{bp}$. In total, we used 113,580,009 100 bp paired-end reads from DL238 ( 227X coverage) and 81,268,657 100 bp paired-end reads from QX1211 ( 163X coverage). We assembled the genomes using SOAPdenovo2 (Luo et al. 2012) with a K-mer size of 23. We then used blastn (Altschul et al. 1990) to search for scaffolds that had homology to sup- 
35, pha-1, and genes in their vicinity. The genomic region that contained the sup-35/pha-1 element and neighboring genes ( $h m t-1$ and Y47D3A.29) was not recovered in a single scaffold because our assemblies lacked mate-pair information. Moreover, the region of interest contained several repetitive elements. To circumvent this limitation and generate a single scaffold, we generated a de novo assembly using an additional software package, DiscovarDenovo (v52488), with default parameters (Weisenfeld et al. 2014). We then used targeted PCR amplification followed by Sanger sequencing to confirm the new scaffolds, and to close remaining gaps. Finally, DL238 and QX1211 Illumina short reads were re-aligned to the assembled haplotypes and visually inspected to discard errors introduced by the assemblers.

\section{Nanopore single molecule sequencing}

We extracted fresh DL238 gDNA using the DNeasy Blood \& Tissue kit (Qiagen) and prepared the sequencing library the same day of extraction following the 1D Genomic DNA by ligation (SQK-LSK108) protocol (Oxford Nanopore Technologies) starting from $1.5 \mu \mathrm{g}$ of gDNA and avoiding DNA fragmentation. Sequencing was performed on a MinION Mk1b using the R9.4 chemistry, and reads were collected for 48 hours. In total, we recovered 194,578 reads that passed quality control (MinKNOW software). Those reads were converted to fastq format using poretools (Loman and Quinlan 2014). To determine whether the long reads supported our de novo assembled DL238 haplotype, we aligned the reads to a modified WBcel235 N2 reference genome that included the DL238 haplotype as an additional scaffold. This allowed us to directly compare the support for either the N2 reference or our de novo assembled haplotype. We aligned the nanopore reads using $b w a$ with the ont $2 d$ flag to allow for the high error rate in the reads. The alignment rate was $94.6 \%$. Average read length was 3,962 bp, and 16,110 reads (11\%) were over $10 \mathrm{~kb}$ in length. 


\section{Multiple sequence alignment}

To study the genomic architecture and evolution of the sup-35/pha-1 element, we recovered the sequence at the sup-35/pha-1 locus in additional Caenorhabditis species. Although the genes themselves are not conserved in any other sequenced nematode species, three genes in close vicinity, hmt-1, Y48A6C.4, and Y47D3A.29, are highly conserved. hmt-1 is a predicted transmembrane $\mathrm{ABC}$ transporter, and $Y 47 D 3 A .29$ encodes the catalytic subunit of DNA polymerase alpha. $h m t-1$ and Y47D3A.29 flank the sup-35/pha-1 element in C. elegans. Y48A6C.4, a predicted ortholog of $S$. cerevisiae IPI1, is located between $h m t-1$ and Y47D3A.29 in all sequenced Caenorhabditis. We took advantage of the high evolutionary conservation of these three genes to recover the sequence of the region in four nematode species (C. briggsae "cb4", $C$. brenneri "6.0.1b", C. remanei "ASM14951v1", and C. angaria "ps1010re14"). Those sequences, together with three C. elegans haplotypes (N2, DL238 and QX1211), were than aligned using progressiveMauve (Darling et al. 2010).

\section{Phylogenetic tree}

The coding sequence of Y48A6C.4 was recovered in the different C. elegans isolates and other Caenorhabditis species. To determine the sequence of the gene in the possible presence of SNVs or indels, we first generated an alternative reference sequence for each of the isolates based on the SNVs and indels identified in our variant calling analysis. We then assembled the cDNA sequences of Y48A6C.4 in each isolate. To that end, we first aligned the sequence around the start and end positions of each exon to the alternative reference using blast (Altschul et al. 1990). Following the alignment, the positions were extracted from the reference and concatenated. This procedure 
allowed us to ensure the correct gene sequence in the presence of SNVs or indels. Alignment of positions from one genome to another using blast is implemented in our liftoveR $\mathrm{R}$ package (https://github.com/eyalbenda/liftoveR). The cDNA sequences of Y48AC6.4 orthologs from other Caenorhabditis were acquired from the Wormbase release WS256. To create a gene tree, the cDNA sequences were in-silico translated, and the protein sequences were aligned using $M A F F T$ (Katoh and Standley 2013). Codon alignment was then generated from the cDNA sequences and the aligned protein sequences using pal2nal (Suyama et al. 2006). Finally, the codon-aligned cDNA sequences were used as input for maximum likelihood phylogenetic reconstruction using MrBayes (4 chains of 1,000,000 cycles) (Ronquist and Huelsenbeck 2003). The general time reversible (GTR) substitution model with gamma distributed rate variation was used as it was found to be the best fit (by minimizing the BIC criterion) using jModelTest2 (Darriba et al. 2012). The resulting tree was visualized using the R package ggtree (Yu et al. 2017). 


\section{References}

Agresti A., Coull B. A., 1998 Approximate is better than "exact" for interval estimation of binomial proportions. Am. Stat. 52: 119-126.

Altschul S. F., Gish W., Miller W., Myers E. W., Lipman D. J., 1990 Basic local alignment search tool. J. Mol. Biol. 215: 403-410.

Andersen E. C., Gerke J. P., Shapiro J. A., Crissman J. R., Ghosh R., Bloom J. S., Félix M.-A., Kruglyak L., 2012 Chromosome-scale selective sweeps shape Caenorhabditis elegans genomic diversity. Nat. Genet. 44: 285-90.

Andersen E. C., Shimko T. C., Crissman J. R., Ghosh R., Bloom J. S., Seidel H. S., Gerke J. P., Kruglyak L., 2015 A Powerful New Quantitative Genetics Platform, Combining Caenorhabditis elegans HighThroughput Fitness Assays with a Large Collection of Recombinant Strains. G3 (Bethesda). 5: $911-$ 20.

Beeman R., Friesen K., Denell R., 1992 Maternal-effect selfish genes in flour beetles. Science 256. Brenner S., 1974 The genetics of Caenorhabditis elegans. Genetics 77: 71-94.

Chen C.-H., Huang H., Ward C. M., Su J. T., Schaeffer L. V., Guo M., Hay B. A., 2007 A Synthetic Maternal-Effect Selfish Genetic Element Drives Population Replacement in Drosophila. Science 316.

Cook D. E., Zdraljevic S., Tanny R. E., Seo B., Riccardi D. D., Noble L. M., Rockman M. V., Alkema M. J., Braendle C., Kammenga J. E., Wang J., Kruglyak L., Félix M.-A., Lee J., Andersen E. C., 2016 The Genetic Basis of Natural Variation in Caenorhabditis elegans Telomere Length. Genetics 204.

Cook D. E., Zdraljevic S., Roberts J. P., Andersen E. C., 2017 CeNDR, the Caenorhabditis elegans natural diversity resource. Nucleic Acids Res. 45: D650-D657.

Darling A. E., Mau B., Perna N. T., 2010 progressiveMauve: multiple genome alignment with gene gain, loss and rearrangement. (JE Stajich, Ed.). PLoS One 5: e11147.

Darriba D., Taboada G. L., Doallo R., Posada D., 2012 jModelTest 2: more models, new heuristics and 
parallel computing. Nat. Methods 9: 772.

Dawkins R., 1976 The Selfish Gene. Oxford University Press.

Doolittle W. F., Sapienza C., 1980 Selfish genes, the phenotype paradigm and genome evolution. Nature 284: $601-603$.

Fay D. S., Qiu X., Large E., Smith C. P., Mango S., Johanson B. L., 2004 The coordinate regulation of pharyngeal development in C. elegans by lin-35/Rb, pha-1, and ubc-18. Dev. Biol. 271: 11-25.

Fay D. S., Polley S. R. G., Kuang J., Kuzmanov A., Hazel J. W., Mani K., Veo B. L., Yochem J., 2012 A Regulatory Module Controlling Pharyngeal Development and Function in Caenorhabditis elegans.

\section{Genetics 191.}

Garrison E., Marth G., 2012 Haplotype-based variant detection from short-read sequencing.

Granato M., Schnabel H., Schnabel R., 1994 Genesis of an organ: molecular analysis of the pha-1 gene. Development 120: 3005-17.

Hahne F., Ivanek R., 2016 Visualizing Genomic Data Using Gviz and Bioconductor. In:, pp. 335-351.

Hammond A., Galizi R., Kyrou K., Simoni A., Siniscalchi C., Katsanos D., Gribble M., Baker D., Marois E., Russell S., Burt A., Windbichler N., Crisanti A., Nolan T., 2015 A CRISPR-Cas9 gene drive system targeting female reproduction in the malaria mosquito vector Anopheles gambiae. Nat. Biotechnol. 34: 78-83.

Hashimshony T., Feder M., Levin M., Hall B. K., Yanai I., 2014 Spatiotemporal transcriptomics reveals the evolutionary history of the endoderm germ layer. Nature 519: 219-222.

Hurst G. D., Werren J. H., 2001 The role of selfish genetic elements in eukaryotic evolution. Nat. Rev. Genet. 2: 597-606.

Katoh K., Standley D. M., 2013 MAFFT multiple sequence alignment software version 7: improvements in performance and usability. Mol. Biol. Evol. 30: 772-80.

Koboldt D. C., Zhang Q., Larson D. E., Shen D., McLellan M. D., Lin L., Miller C. A., Mardis E. R., Ding L., Wilson R. K., 2012 VarScan 2: Somatic mutation and copy number alteration discovery in cancer by exome sequencing. Genome Res. 22: 568-576. 
Kuzmanov A., Yochem J., Fay D. S., 2014 Analysis of PHA-1 Reveals a Limited Role in Pharyngeal Development and Novel Functions in Other Tissues. Genetics 198.

Langmead B., Trapnell C., Pop M., Salzberg S. L., 2009 Ultrafast and memory-efficient alignment of short DNA sequences to the human genome. Genome Biol. 10: R25.

Larracuente A. M., Presgraves D. C., 2012 The Selfish Segregation Distorter Gene Complex of Drosophila melanogaster. Genetics 192.

Li H., Durbin R., 2010 Fast and accurate long-read alignment with Burrows-Wheeler transform. Bioinformatics 26: 589-595.

Loman N. J., Quinlan A. R., 2014 Poretools: a toolkit for analyzing nanopore sequence data. Bioinformatics 30: 3399-401.

Lorenzen M. D., Gnirke A., Margolis J., Garnes J., Campbell M., Stuart J. J., Aggarwal R., Richards S., Park Y., Beeman R. W., 2008 The maternal-effect, selfish genetic element Medea is associated with a composite Tc1 transposon. Proc. Natl. Acad. Sci. U. S. A. 105: 10085-9.

Luo R., Liu B., Xie Y., Li Z., Huang W., Yuan J., He G., Chen Y., Pan Q., Liu Y., Tang J., Wu G., Zhang H., Shi Y., Liu Y., Yu C., Wang B., Lu Y., Han C., Cheung D. W., Yiu S.-M., Peng S., Xiaoqian Z., Liu G., Liao X., Li Y., Yang H., Wang J., Lam T.-W., Wang J., 2012 SOAPdenovo2: an empirically improved memory-efficient short-read de novo assembler. Gigascience 1: 18.

Mango S. E., 2009 The Molecular Basis of Organ Formation: Insights From the C. elegans Foregut.

Annu. Rev. Cell Dev. Biol. 25: 597-628.

Mani K., Fay D. S., 2009 A Mechanistic Basis for the Coordinated Regulation of Pharyngeal Morphogenesis in Caenorhabditis elegans by LIN-35/Rb and UBC-18-ARI-1 (SK Kim, Ed.). PLoS Genet. 5: e1000510.

McKenna A., Hanna M., Banks E., Sivachenko A., Cibulskis K., Kernytsky A., Garimella K., Altshuler D., Gabriel S., Daly M., DePristo M. A., 2010 The Genome Analysis Toolkit: a MapReduce framework for analyzing next-generation DNA sequencing data. Genome Res. 20: 1297-303.

Okkema P. G., Ha E., Haun C., Chen W., Fire A., 1997 The Caenorhabditis elegans NK-2 homeobox 
gene ceh-22 activates pharyngeal muscle gene expression in combination with pha-1 and is required for normal pharyngeal development. Development 124.

Orgel L. E., Crick F. H. C., 1980 Selfish DNA: the ultimate parasite. Nature 284: 604-607.

Pollard K. S., Hubisz M. J., Rosenbloom K. R., Siepel A., 2010 Detection of nonneutral substitution rates on mammalian phylogenies. Genome Res. 20: 110-21.

Polley S. R. G., Kuzmanov A., Kuang J., Karpel J., Lažetić V., Karina E. I., Veo B. L., Fay D. S., 2014

Implicating SCF Complexes in Organogenesis in Caenorhabditis elegans. Genetics 196.

Rimmer A., Phan H., Mathieson I., Iqbal Z., Twigg S. R. F., WGS500 Consortium A. O. M., Wilkie A. O. M., McVean G., Lunter G., 2014 Integrating mapping-, assembly- and haplotype-based approaches for calling variants in clinical sequencing applications. Nat. Genet. 46: 912-8.

Ronquist F., Huelsenbeck J. P., 2003 MrBayes 3: Bayesian phylogenetic inference under mixed models. Bioinformatics 19: 1572-4.

Schedl T., Kimble J., 1988 fog-2, a germ-line-specific sex determination gene required for hermaphrodite spermatogenesis in Caenorhabditis elegans. Genetics 119.

Schendel R. van, Roerink S. F., Portegijs V., Heuvel S. van den, Tijsterman M., Hoeijmakers J. H., Wang H., Wilson T. E., Grawunder U., Lieber M. R., Boulton S. J., Jackson S. P., Ceccaldi R., Koole W., Mateos-Gomez P. A., McVey M., Lee S. E., Roerink S. F., van S. R., Tijsterman M., Yousefzadeh M. J., Yoon J. H., Roy C. J., Park J., Prakash S., Prakash L., Seki M., Shima N., Munroe R. J., Schimenti J. C., Fernandez-Vidal A., Shima N., Kloosterman W. P., Villarreal D. D., AshwoodSmith M. J., Edwards R. G., Hamer G., Robert V., Bessereau J. L., Plasterk R. H., Robert V. J., Davis M. W., Jorgensen E. M., Bessereau J. L., Grishkevich V., Thompson O., Sijen T., Plasterk R. H., Yu A. M., McVey M., Chan S. H., Yu A. M., McVey M., Jinek M., Waaijers S., Gratz S. J., Hwang W. Y., Mali P., Clejan I., Boerckel J., Ahmed S., Lemmens B. B., Johnson N. M., Tijsterman M., Adamo A., Kent T., Chandramouly G., McDevitt S. M., Ozdemir A. Y., Pomerantz R. T., Carvalho C. M., Boeva V., Higgins G. S., Brenner S., Mori I., Moerman D. G., Waterston R. H., Dickinson D. J., Ward J. D., Reiner D. J., Goldstein B., Tzur Y. B., Li H., Durbin R., Li H., 
McKenna A., Ye K., Schulz M. H., Long Q., Apweiler R., Ning Z., Thorvaldsdottir H., Robinson J. T., Mesirov J. P., Andersen E. C., Keane T. M., Wong K., Adams D. J., Purcell S., Lee T. H., Guo H., Wang X., Kim C., Paterson A. H., Ketting R. F., Haverkamp T. H., Luenen H. G. van, Plasterk R. H., 2015 Polymerase $\Theta$ is a key driver of genome evolution and of CRISPR/Cas9-mediated mutagenesis. Nat. Commun. 6: 7394.

Schnabel H., Schnabel R., 1990 An Organ-Specific Differentiation Gene, pha-1, from Caenorhabditis elegans. Science 250.

Schnabel H., Bauer G., Schnabel R., 1991 Suppressors of the organ-specific differentiation gene pha-1 of Caenorhabditis elegans. Genetics 129.

Seidel H. S., Rockman M. V, Kruglyak L., 2008 Widespread genetic incompatibility in C. elegans maintained by balancing selection. Science 319: 589-94.

Seidel H. S., Ailion M., Li J., Oudenaarden A. van, Rockman M. V, Kruglyak L., 2011 A novel spermdelivered toxin causes late-stage embryo lethality and transmission ratio distortion in C. elegans (LD Hurst, Ed.). PLoS Biol. 9: e1001115.

Shin H.-S., Flaherty L., Artzt K., Bennett D., Ravetch J., 1983 Inversion in the H-2 complex of thaplotypes in mice. Nature 306: 380-383.

Sinkins S. P., 2011 Invertebrate Post-Segregation Distorters: A New Embryo-Killing Gene. PLoS Biol. 9: e1001114.

Suyama M., Torrents D., Bork P., 2006 PAL2NAL: robust conversion of protein sequence alignments into the corresponding codon alignments. Nucleic Acids Res. 34: W609-12.

Weisenfeld N. I., Yin S., Sharpe T., Lau B., Hegarty R., Holmes L., Sogoloff B., Tabbaa D., Williams L., Russ C., Nusbaum C., Lander E. S., MacCallum I., Jaffe D. B., 2014 Comprehensive variation discovery in single human genomes. Nat. Genet. 46: 1350-1355.

Werren J. H., 1997 Biology of Wolbachia. Annu. Rev. Entomol. 42: 587-609.

Werren J. H., 2011 Selfish genetic elements, genetic conflict, and evolutionary innovation. Proc. Natl. Acad. Sci. U. S. A.: 10863-70. 
Wickham H., 2009 ggplot2: Elegant Graphics for Data Analysis (Use R!). Springer.

Yu G., Smith D. K., Zhu H., Guan Y., Lam T. T.-Y., 2017 ggtree : an r package for visualization and annotation of phylogenetic trees with their covariates and other associated data (G McInerny, Ed.). Methods Ecol. Evol. 8: 28-36. 


\section{Figures}

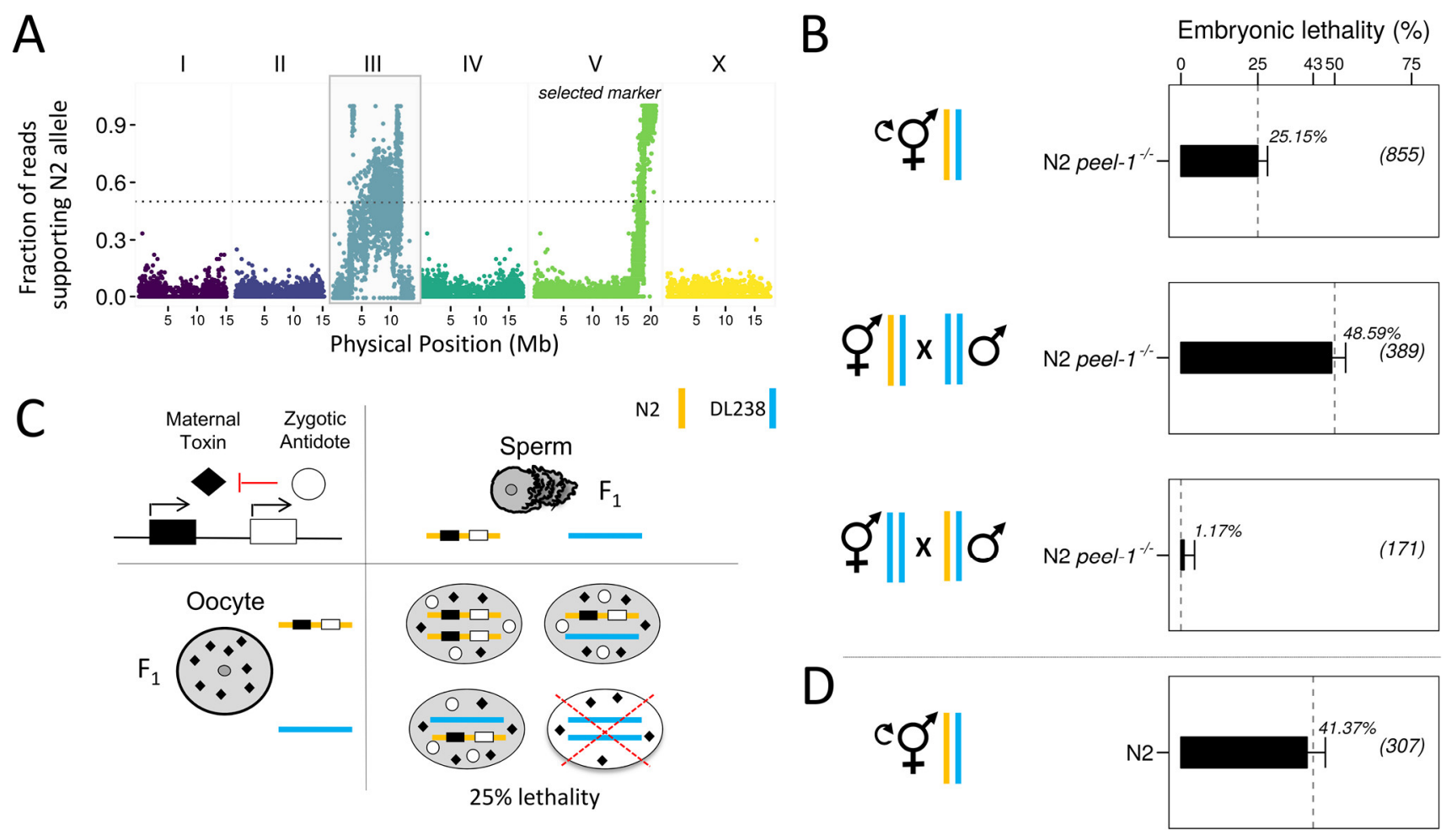

Figure 1. A maternal-effect genetic incompatibility on Chr. III. (A) A marker on Chr. V was introgressed from the reference strain N2 into the DL238 wild isolate. Short-read sequencing of the introgression strain revealed homozygous N2 variants on Chr. III, indicating strong selection in favor of $\mathrm{N} 2$ variants during the generation of this strain. (B) DL238 males were crossed to hermaphrodites carrying a null allele of the peel-1/zeel-1 element (niDf9) in an otherwise $\mathrm{N} 2$ background (N2 peel-1 $1^{--}$). $\mathrm{F}_{1}$ hermaphrodites were allowed to self-fertilize (top). Alternatively, $F_{1}$ males (middle) or hermaphrodites (bottom) were backcrossed to the DL238 parental strain. Embryonic lethality was scored in the $\mathrm{F}_{2}$ progeny as percent of unhatched eggs. Dashed grey lines indicate expected embryonic lethality under the maternaleffect toxin and zygotic antidote model (see also Fig. S2). Sample sizes are shown in parentheses. Error bars indicate $95 \%$ binomial confidence intervals, calculated using the Agresti-Coull method (Agresti and Coull 1998) (C) Punnett square showing the expected lethality in the $F_{2}$. An interaction between a maternal toxin (black rhombus) and a zygotic antidote (white circle) results in $25 \%$ embryonic lethality in the $\mathrm{F}_{2}$ and is compatible with the lethality observed in our crosses. (D) Embryonic lethality in the $\mathrm{F}_{2}$ progeny of a cross between wild-type N2 hermaphrodites and DL238 males. N2 carries an active copy of peel-1/zeel-1, while DL238 carries an inactive copy. Independent segregation of two fully penetrant parental-effect incompatibilities is expected to result in $43.75 \%$ embryonic lethality. Orange and blue bars denote N2 and DL238 haplotypes, respectively. 


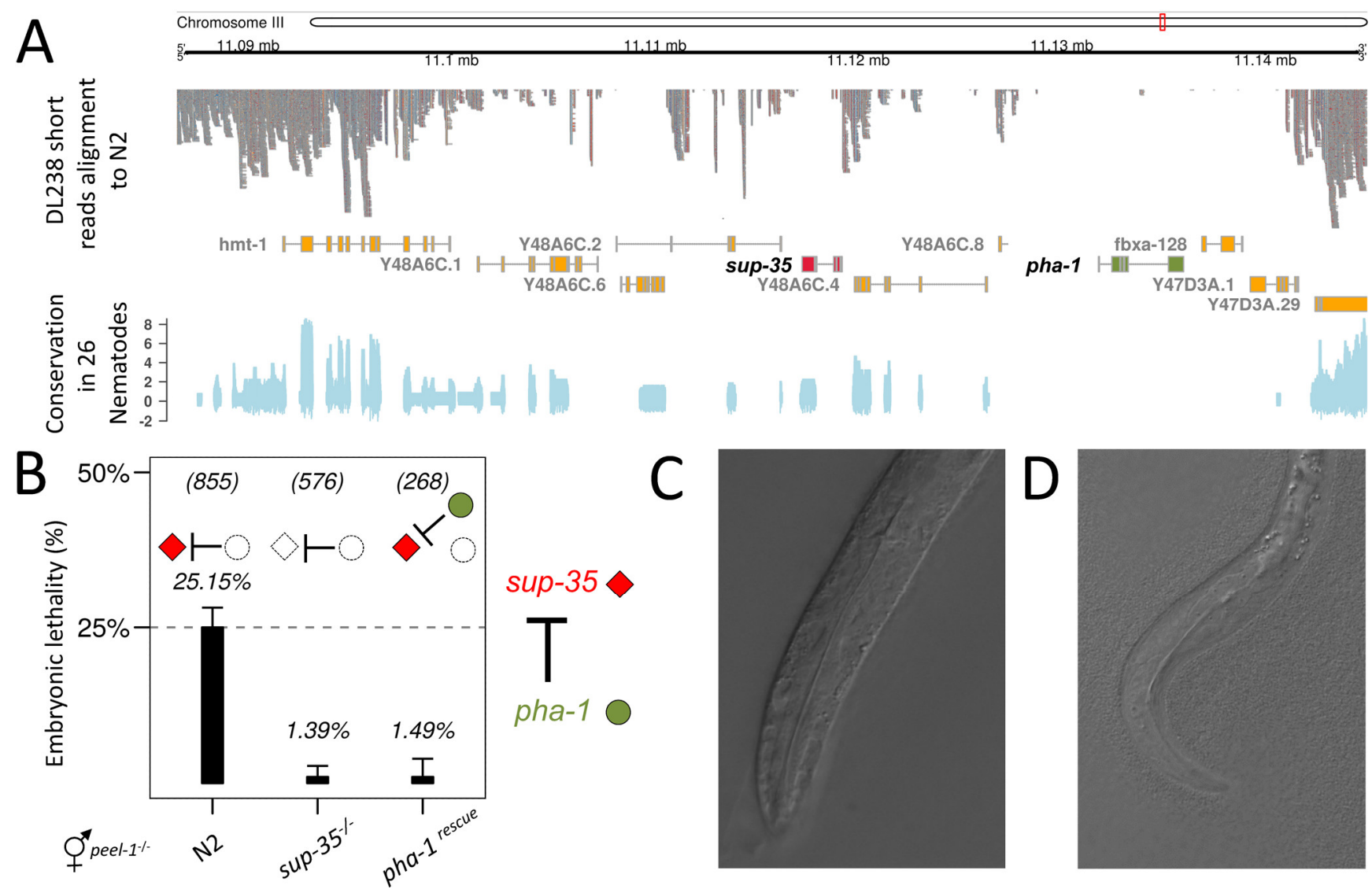

Figure 2. sup-35 and pha-1 encode a maternal-effect genetic incompatibility. (A) Alignment of short reads from DL238 to the N2 reference genome (top). A $\sim 50 \mathrm{~kb}$ region on the right arm of Chr. III selected during the introgression shows sparse alignment throughout with no read support for pha-l (green) and weak support for sup-35 (red). Sequence conservation across 26 nematodes showed no conservation of pha1 (bottom). Values are phyloP scores retrieved from the UCSC genome browser (Pollard et al. 2010) (B) In our model, sup-35 (red rhombus) is a maternally deposited toxin and pha-1 is a zygotically expressed antidote (green circle). The embryonic lethality in the $\mathrm{F}_{2}$ of the cross between DL238 and N2 peel-1/- (left) was completely rescued when DL238 males were crossed to a strain carrying a sup-35(e2223) loss of function allele (center), and also when both parents carried a pha-1 transgene (right). Error bars indicate 95\% binomial confidence intervals, calculated using the Agresti-Coull method (Agresti and Coull 1998) (C) The pharynx of a phenotypically wild-type $\mathrm{F}_{2} \mathrm{~L} 1$ worm from a DL238 x N2 peel- $1^{-/}$cross. (D) The pharynx of an $F_{2} L 1$ from the same cross as in (C) showing pharyngeal morphological defects and arrested development. 


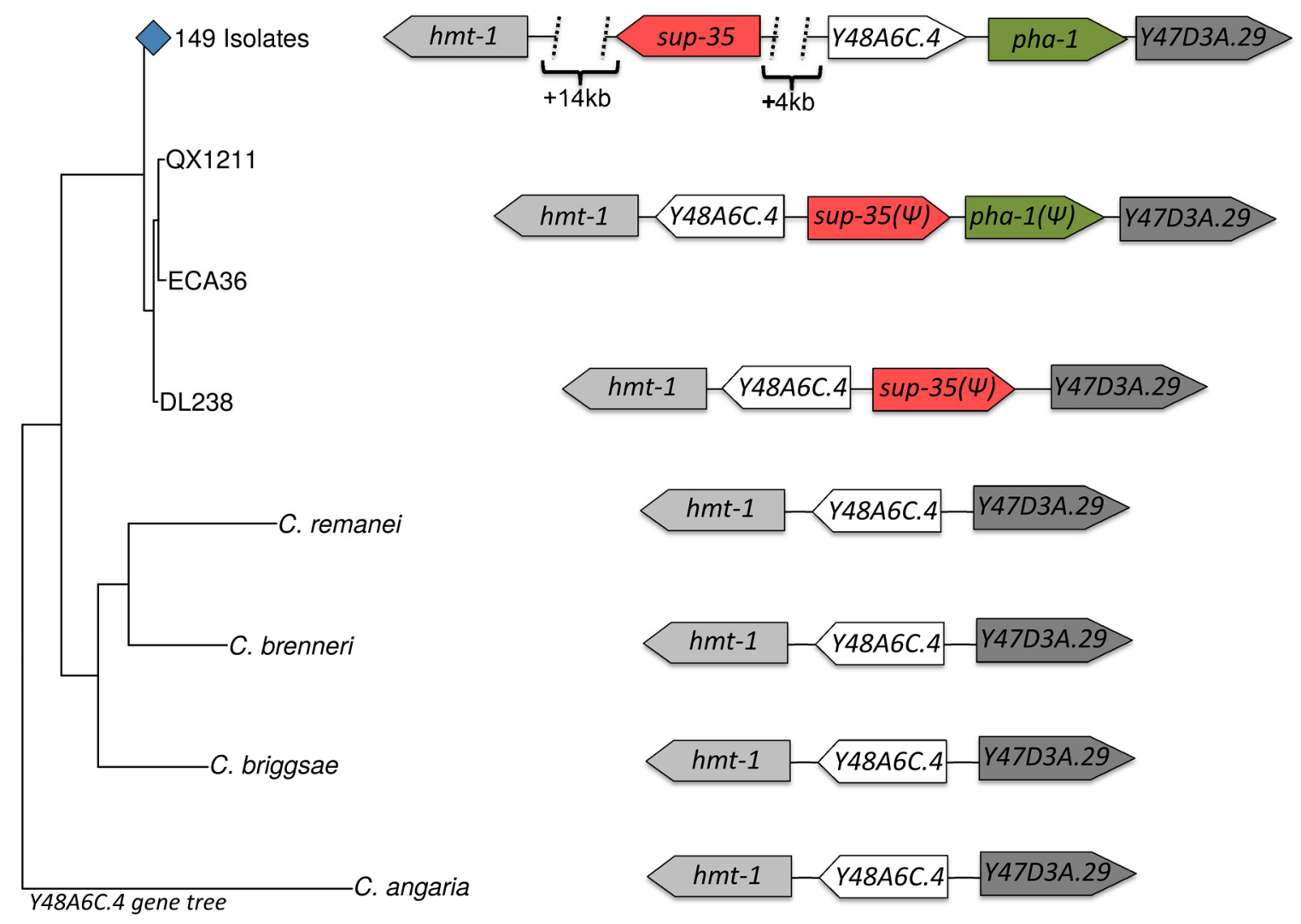

Figure 3. The sup-35/pha-1 N2 haplotype is derived and is marked by an inversion. (Left) A gene tree built from the coding region of Y48A6C.4 in 152 C. elegans isolates and four other Caenorhabditis species. DL238, QX1211 and ECA36 cluster together in a separate branch from all other C. elegans isolates. (Right) The synteny in the region containing the sup-35/pha-1 element, as well as three highly conserved genes in the close vicinity ( $h m t-1, Y 48 A 6 C .4$, and Y47D3A.29) is schematically represented. ( $\psi$ ) denotes alleles that are pseudogenized. The genes sup-35 (red) and Y48A6C.4 (white) are inverted in DL238, QX1211, and ECA36 relative to the other 149 C. elegans isolates. The gene order and orientation of hmt-1, Y48A6C.4, and Y47D3A.29 in other Caenorhabditis species suggests that the inverted haplotype is the ancestral, and that the haplotype found in 149 isolates is the derived one. 


\section{Supplementary Figures}

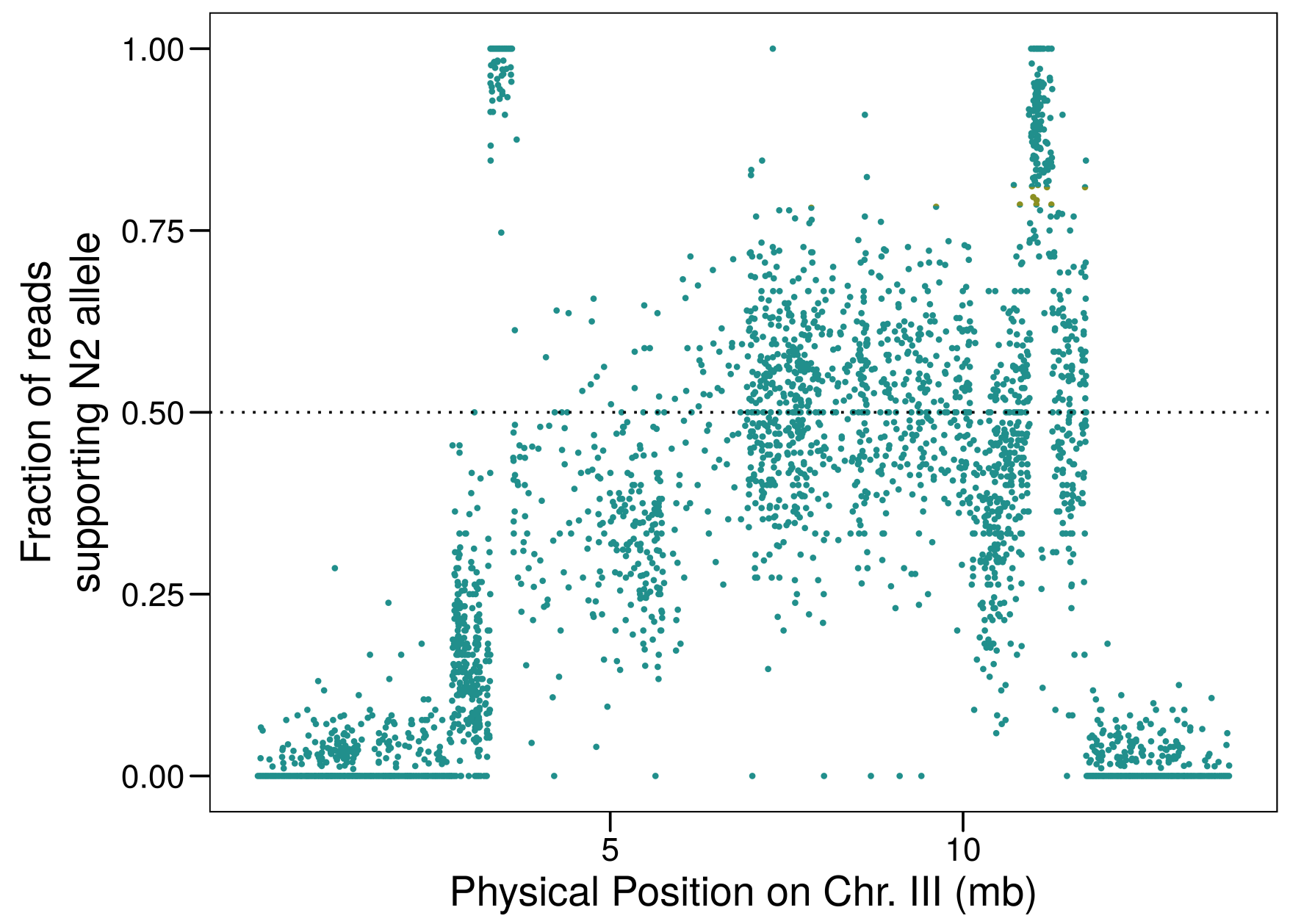

Figure S1. Selection for N2 alleles on Chr. III when introgressing a genetic marker on Chr. $V$ from $\mathbf{N 2}$ into DL238. Each point is an SNV that was identified in DL238. A strict pipeline was used to reduce the incidence of spurious SNVs (see Materials and Methods). We identified two regions homozygous for N2 alleles: Chr III. 3,302,436-3,607,723 and Chr. III. 10,964,134$11,254,095$. The region closer to the right arm shows linkage decay, suggesting it harbors the locus under selection. 

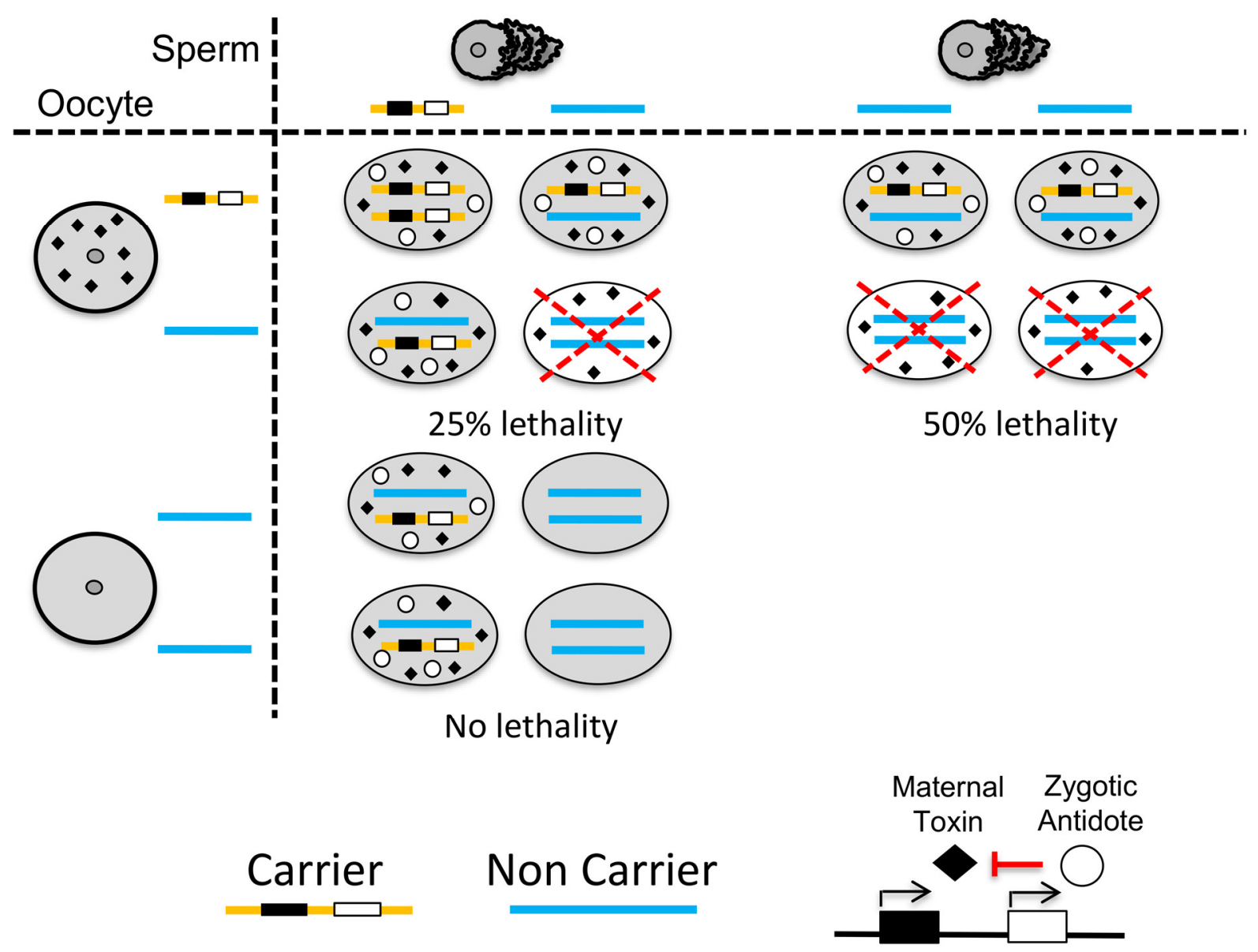

Figure S2. Expected $F_{2}$ embryonic lethality due to the action of a maternal-effect toxin linked to its zygotic antidote. The selfish element (shown on the bottom right) is encoded by two tightlylinked genes: a maternal-effect toxin (black rhombus) and a zygotic antidote (white circle). In a cross between a carrier (orange) and a non-carrier (blue), all $\mathrm{F}_{1}$ will carry one copy of the element. When these $F_{1}$ self (top left), all of their progeny inherit the maternal-effect toxin, regardless of their genotype. However, $25 \%$ of the progeny will not inherit a copy of the antidote, leading to lethality. A maternal-effect toxin can be distinguished from a paternal-effect toxin by backcrossing the $F_{1}$ to non-carriers. When crossing $F_{1}$ hermaphrodites to non-carrier males (top right), all of the progeny inherit the maternal-effect toxin, but 50\% do not inherit the antidote and die. When the reciprocal backcross is done, none of the progeny inherit the toxin by maternal-effect, so all progeny that carry the toxin also carry the antidote, and no lethality is observed. 


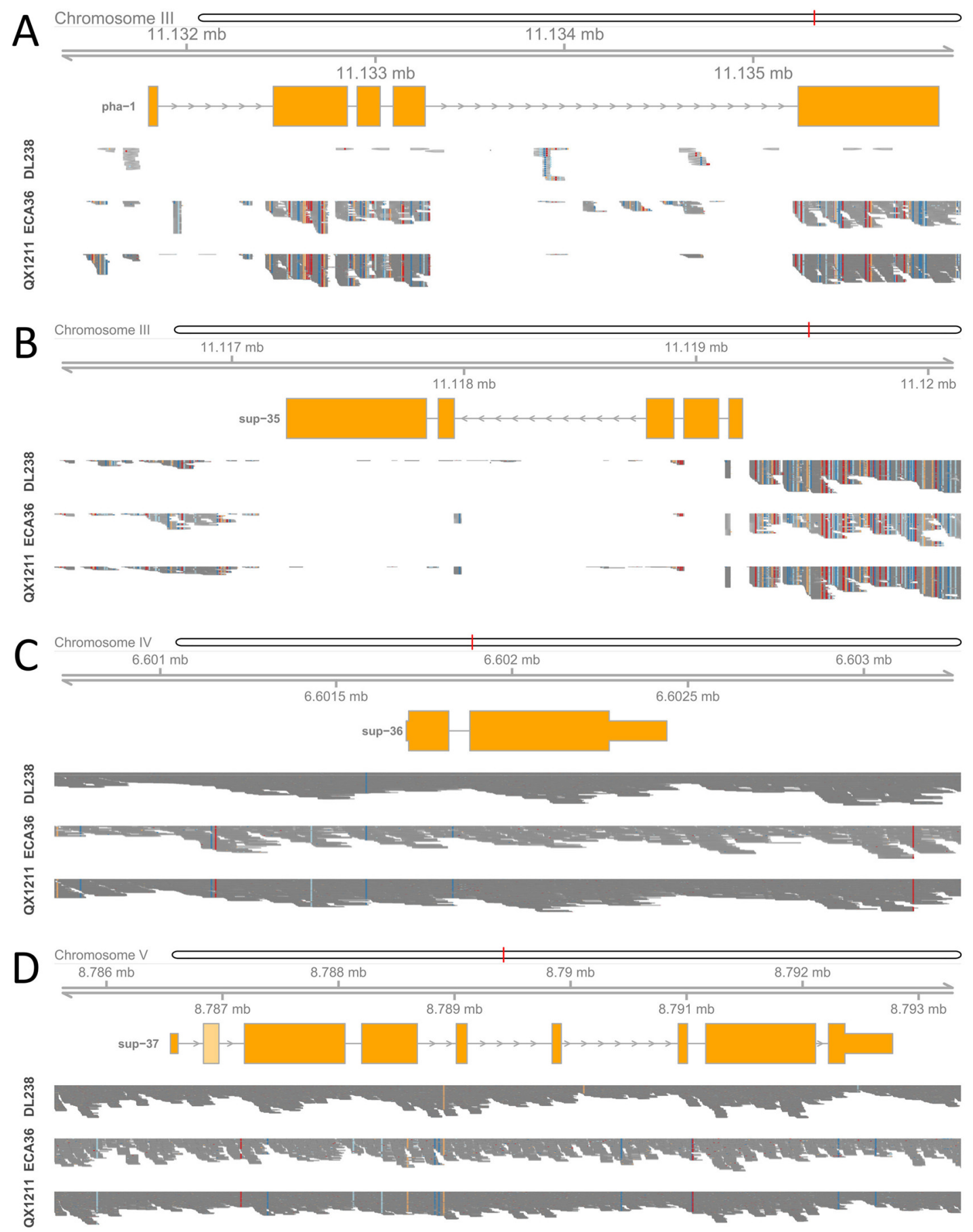

Figure S3. Alignment of short reads from DL238, ECA36 and QX1211 to the N2 reference in pha-1, sup-35, sup-36, and sup-37. Illumina short reads from DL238, ECA36, and QX1211 wild isolates were aligned against the $\mathrm{N} 2$ reference genome. A pileup of the reads aligning to pha1 (A). sup-35 (B), sup-36 (C) and sup-37 (D) is shown. Gray bars illustrate a match and colors indicate mismatches. Lack of alignment strongly suggests that a gene is missing or that the sequence is highly divergent compared to N2. 


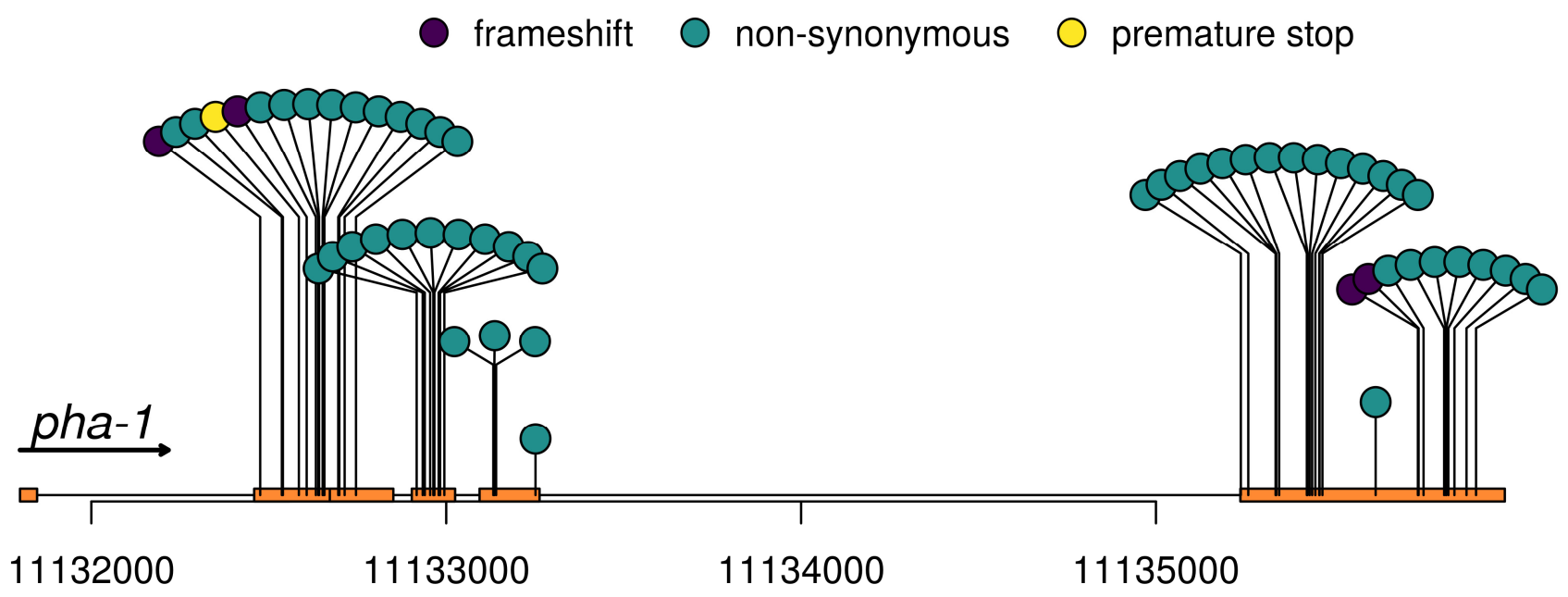

Figure S4. Variants in pha-1 in the QX1211 and ECA36 wild isolates. pha-1 appears present in QX1211 and ECA36 but has accumulated a large number of mutations compared to the reference N2. The large accumulation of coding variants, including frameshifts, strongly suggests that pha-1 underwent pseudogenization in QX1211 and ECA36. 
bioRxiv preprint doi: https://doi.org/10.1101/112524; this version posted March 1, 2017. The copyright holder for this preprint (which was not certified by peer review) is the author/funder, who has granted bioRxiv a license to display the preprint in perpetuity. It is made available under aCC-BY-NC-ND 4.0 International license.

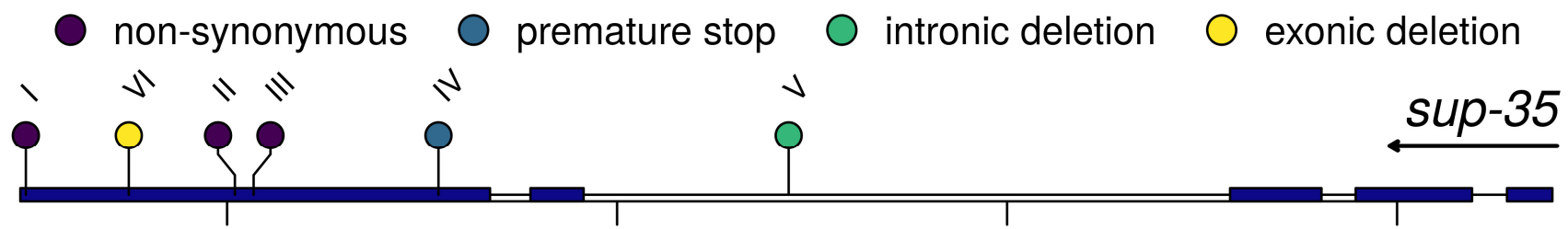

11117500

11118000

11118500

11119000

\begin{tabular}{ccccccc}
\hline & chr & position & Ref & Alt & cDNA & protein \\
\hline I & III & 11117242 & G & A & c.992C $>$ T & p.Ser331Leu \\
\hline II & III & 11117510 & A & T & c.724T $>$ A & p.Ser242Thr
\end{tabular}

AB1, CB4852, ED3005, EG4347, JT11398, JU1395, JU1896, JU2513, MY18, WN2002

AB1, CB4852, CB4854, CB4856, CX11254, CX11315, ED3005, ED3040, EG4347, EG4349, EG4724, EG4725, GXW1, JT11398, JU1242, JU1395, JU1400, JU1409, JU1440, JU1491, JU1530, JU1581, JU1652, JU1896, JU2001, JU2513, JU2519, JU2522, JU2526, JU310, JU346, JU360, JU561, JU751, JU774, JU775, JU778, JU782, JU792, JU830, LKC34, MY1, MY18, NIC1, NIC166, NIC195, NIC236, NIC256, NIC258, NIC259, NIC260, NIC261, NIC262, NIC265, NIC267, NIC268, NIC271, NIC272, NIC276, NIC277, NIC3, QX1792, QX1793, RC301, WN2001, WN2002

\begin{tabular}{|c|c|c|c|c|c|c|c|c|c|}
\hline III & III & 11117534 & $\mathrm{~T}$ & G & c. $700 \mathrm{~A}>\mathrm{C}$ & p.Asn234His & $\begin{array}{l}\text { AB1, CB4852, CB4854, CB4856, CX11254, } \\
\text { CX11315, ED3005, ED3017, ED3040, } \\
\text { ED3073, EG4347, EG4349, EG4724, } \\
\text { EG4725, GXW1, JT11398, JU1242, JU1246, } \\
\text { JU1400, JU1409, JU1440, JU1491, JU1530, } \\
\text { JU1580, JU1581, JU1652, JU1896, JU2001, } \\
\text { JU2316, JU2513, JU2519, JU2522, JU2526, } \\
\text { JU258, JU310, JU346, JU360, JU561, JU751, } \\
\text { JU774, JU775, JU778, JU782, JU792, JU830, } \\
\text { LKC34, MY1, MY10, MY18, NIC1, NIC166, } \\
\text { NIC236, NIC251, NIC256, NIC258, NIC259, } \\
\text { NIC260, NIC261, NIC262, NIC265, NIC267, } \\
\text { NIC268, NIC271, NIC272, NIC277, NIC3, } \\
\text { QW947, QX1793, RC301, WN2001, } \\
\text { WN2002 }\end{array}$ & $\begin{array}{l}\text { JT11398, } \\
\text { CB4856, } \\
\text { JU1652 }\end{array}$ & Yes \\
\hline IV & III & 11117771 & $\mathrm{C}$ & A & c. $463 \mathrm{G}>\mathrm{T}$ & p.Gly $155^{*}$ & JU1400 & JU1400 & No \\
\hline $\mathbf{V}$ & III & 11118220 & · & $\sim 600$ bp deletion & & . & $\begin{array}{l}\text { CB4854, CX11254, EG4349, GXW1, } \\
\text { JU1530, JU2007, JU310, JU360, JU751, } \\
\text { JU775, JU830, MY1, NIC199, NIC252, } \\
\text { NIC256, NIC258, NIC260, NIC261, NIC262, } \\
\text { NIC267, NIC269, NIC271, NIC277, NIC3, } \\
\text { QW947, RC301 }\end{array}$ & MY1 & Yes \\
\hline VI & III & 11117374 & . & $\sim 12.1 \mathrm{~kb}$ deletion & . & . & $\begin{array}{l}\text { BRC20067, ED3012, JU2007, NIC199, } \\
\text { NIC252, NIC255, NIC269 }\end{array}$ & $\begin{array}{c}\text { BRC20067, } \\
\text { ED3012, } \\
\text { NIC199 }\end{array}$ & No \\
\hline
\end{tabular}

Figure S5. Coding and structural variants in sup-35 across 152 C. elegans wild isolates. DL238, QX1211, and ECA36, which carry a pseudogenized copy of sup-35 were excluded from the analysis. For each of the variants, we tested carriers by crossing them with DL238. In carriers of two variants (IV, VI), the maternal-effect incompatibility was abolished in the cross, indicating they are null sup-35 alleles. For the intronic deletion (V), the center of the deletion is marked. For the large $12 . \mathrm{kb}$ deletion that includes part of the last exon of sup-35, the marker shows the position from which point sup35 is deleted. 


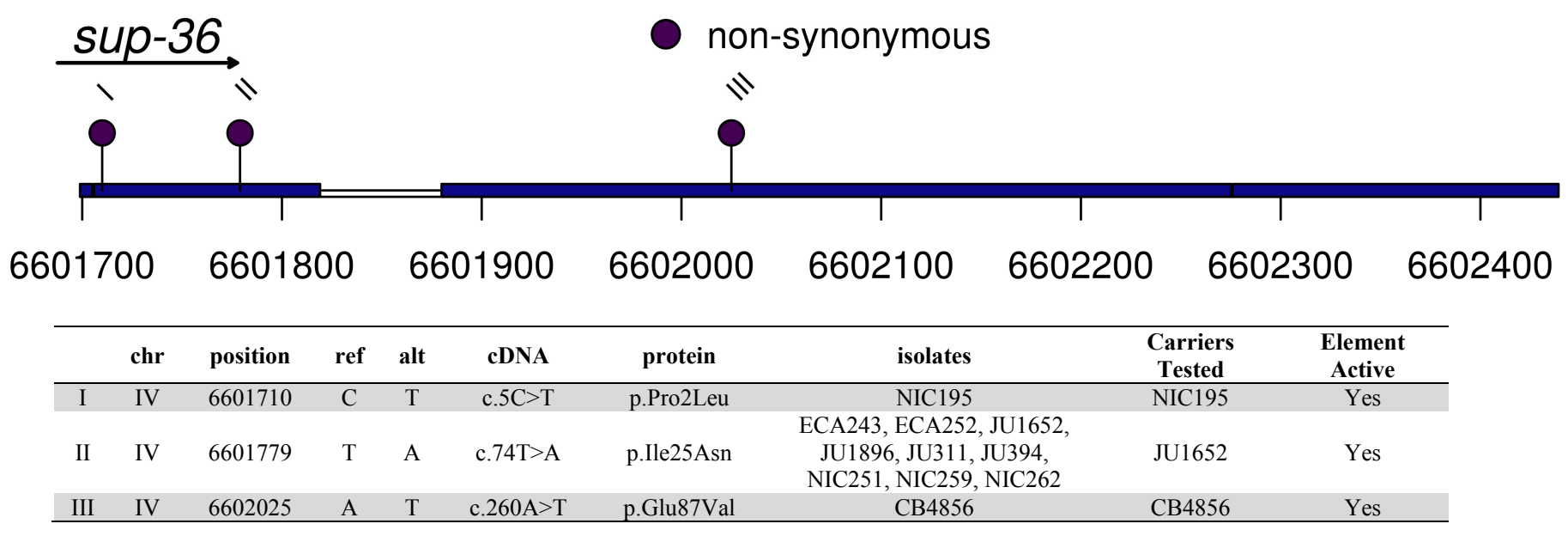

Figure S6. Coding variants in sup-36 across 152 wild C. elegans wild isolates. Isolates carrying each variant were tested by crossing to DL238, and no evidence was found for reduced lethality. 


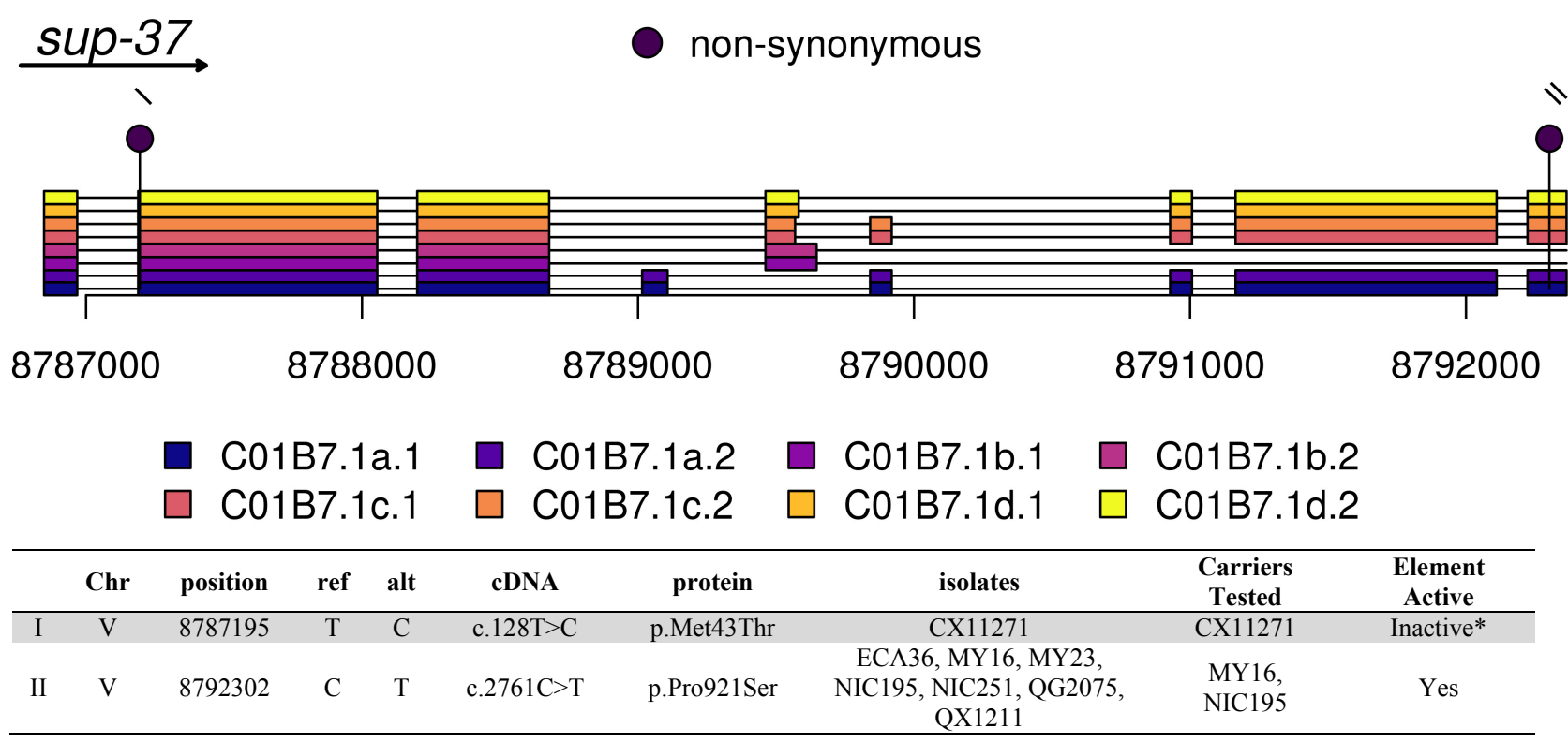

Figure S7. Coding variants in sup-37 across 152 wild C. elegans wild isolates. Isolates carrying each variant were tested by crossing to DL238.

*The isolate CX11271 (Pasadena, CA, USA) that carries the p.Met43Thr (I) variant showed reduced embryonic lethality when crossed to DL238 (17\% in the $\left.\mathrm{F}_{2}\right)$. This lethality was significantly different from the expected $25 \%\left(p=4.9 \times 10^{-7}\right.$, binomial test, CI: $\left.14.25 \%-20.00 \%\right)$ and consistent with the expected $18.75 \%$ if this variant behave like a fully penetrant recessive suppressor (see Materials and Methods for a derivation of the expected lethality). Genotyping of $15 \mathrm{~F}_{2}$ dead embryos identified 10 that were heterozygote $(\mathrm{C} / \mathrm{T}), 5$ that were homozygote for the wild-type $(\mathrm{T} / \mathrm{T})$ allele, but no $(\mathrm{C} / \mathrm{C})$ homozygotes for the $\mathrm{CX} 11271$ variant $(p=0.03$, binomial test), giving further support to the observation that this variant abolishes sup-35 lethality. 
A

Y48A6C.1, a chimeric pseudogene

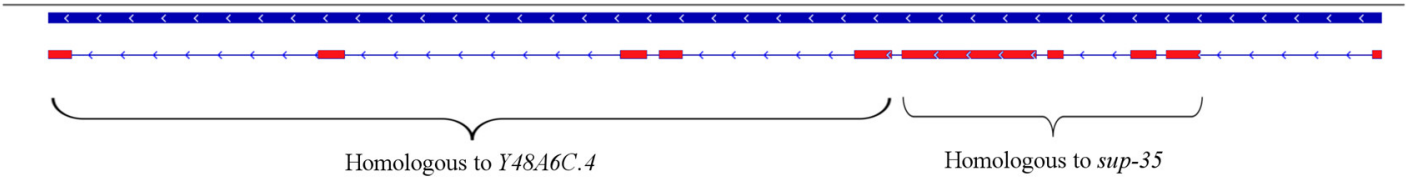

B

Chr. III

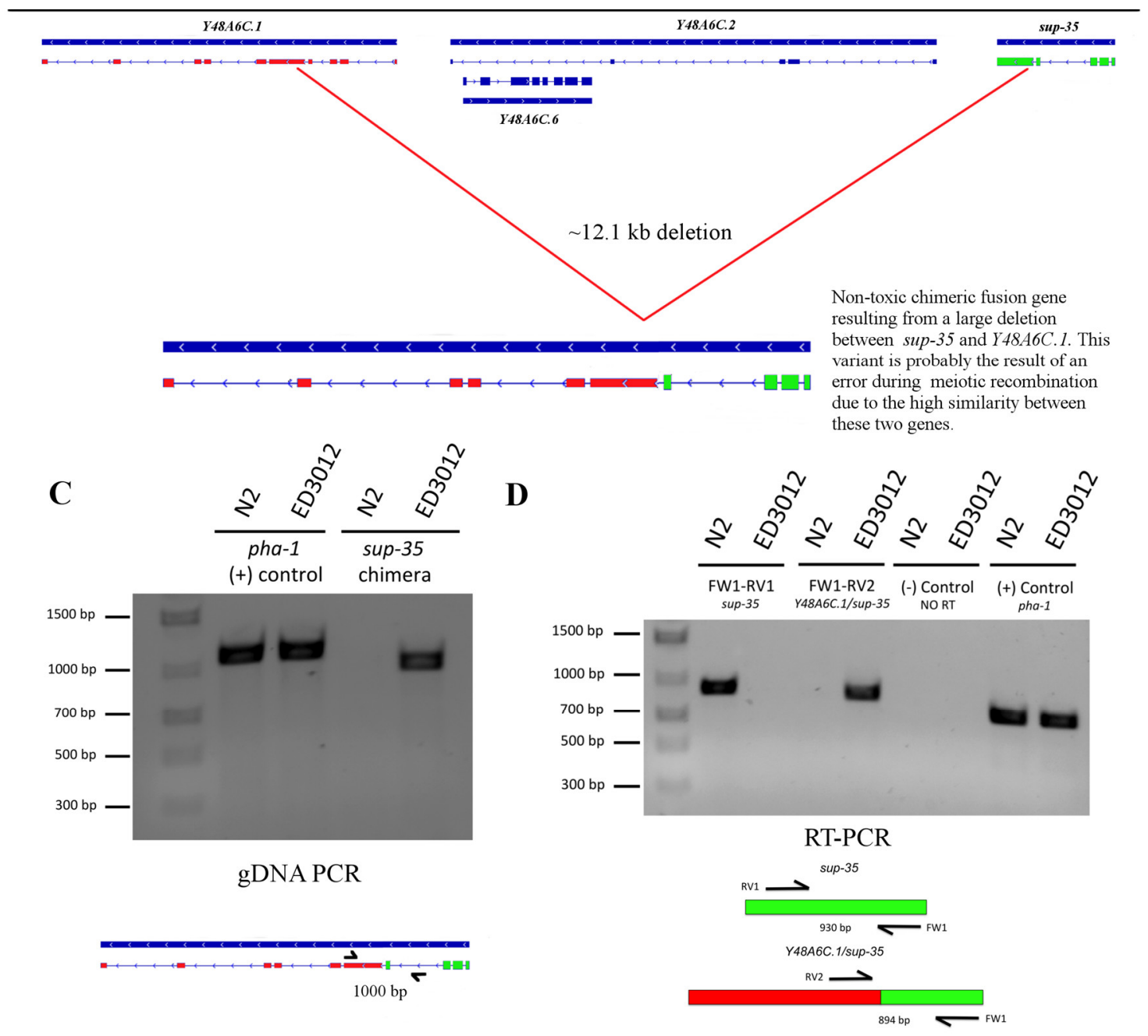

Figure S8. A large deletion identified in seven wild isolates results in a non-functional chimeric transcript that fuses sup-35 and Y48A6C.1. (A) Y48A6C.1 is a pseudogene, which has four exons that are homologous to sup-35 and five that are homologous to Y48A6C.4. (B) We identified 7 isolates (fig. S4) that carry a $12.1 \mathrm{~kb}$ deletion that fuses sup-35 to Y48A6C.1. (C) Primers flanking the $12.1 \mathrm{~Kb}$ deletion amplify the expected amplicon in ED3012, a wild isolate carrying the deletion, but not in N2. (D) Reversetranscription PCR (RT-PCR) from N2 and ED3012 worms shows that the chimeric Y48A6C.1/sup-35 fusion gene is expressed and spliced. FW1/RV1 primer pairs are specific for the WT sup-35 and FW1/RV2 are specific for the sup-35/Y48A6C.1 fusion (FW1-RV2). Primer FW1 hybridizes in an exon-exon junction in sup-35. The RT enzyme was not added to the reaction as a negative control. pha-l expression was used as a positive control. 


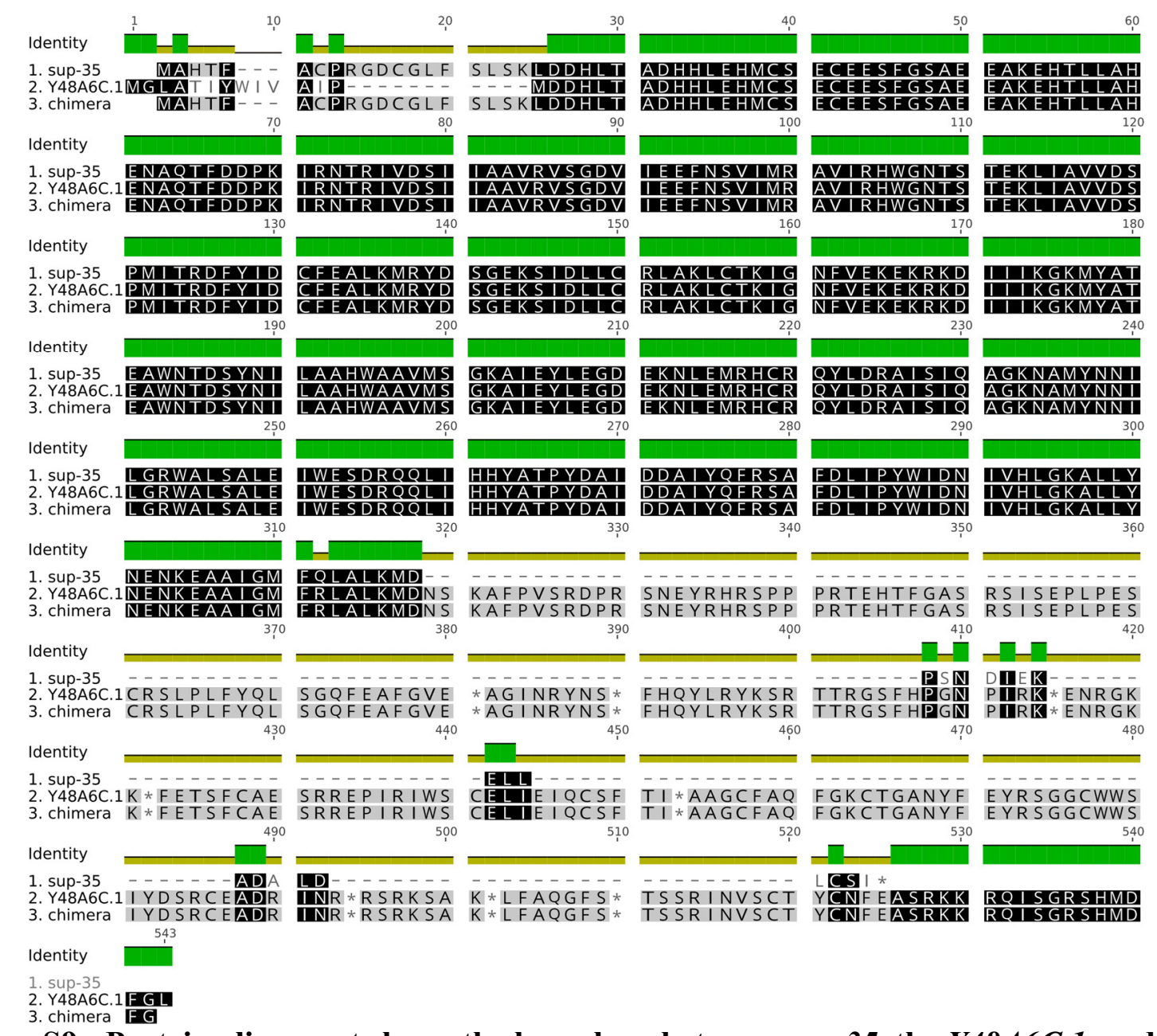

Figure S9: Protein alignment shows the homology between sup-35, the $Y 48$ A6C.1, and the chimeric gene that results from a deletion identified in seven wild isolates. Darker colors denote higher conservation. Similarly, conservation is also indicated by the color identity bar on top (red: low conservation, green: complete conservation. yellow: moderate conservation). 


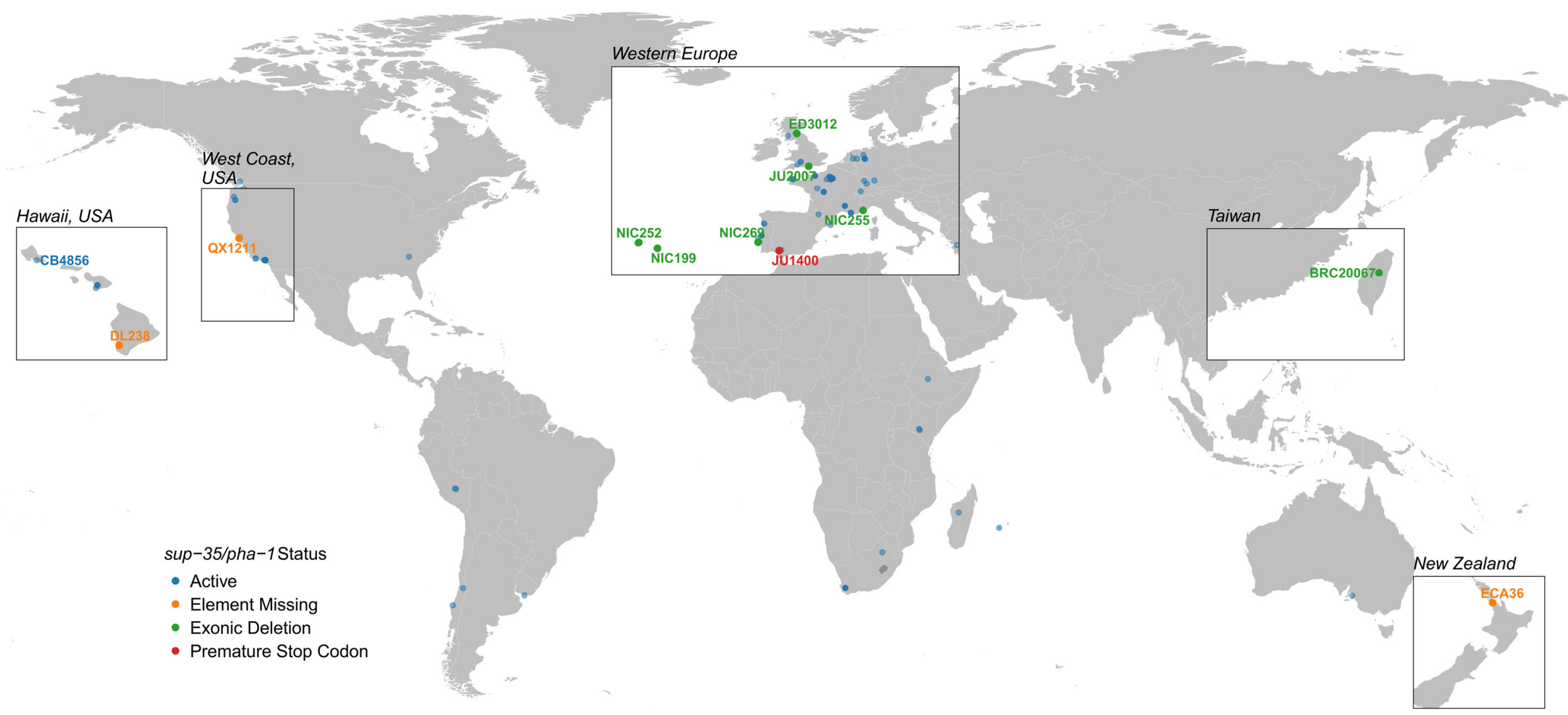

Figure S10. Worldwide distribution of sup-35/pha-1 activity across 152 isolates. Each circle represents a single isolate. Only those isolates with an inactive sup-35/pha-1 element are named, with the exception of CB4856, a highly divergent isolate from Hawaii, which nevertheless carries the active element. 


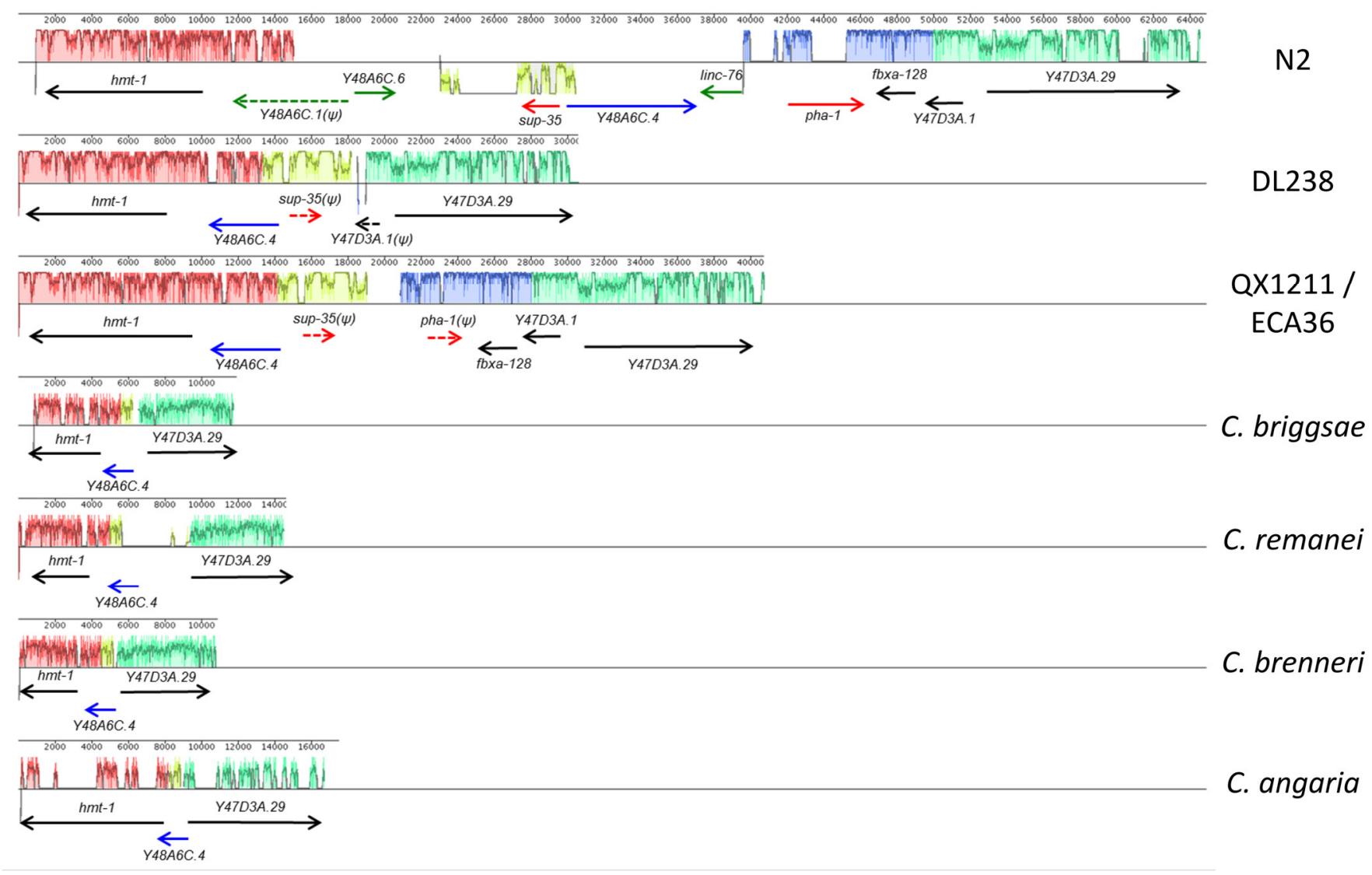

Figure S11. Multiple sequence alignment of the region containing the sup-35/pha-1 element across $C$. elegans isolates and Caenorhabditis species. Two genes, hmt-1 and Y47D3A.29, flank the sup-35/pha-1 element in the N2 reference genome. Because these two genes are highly conserved in the Caenorhabditis genus, we performed a multiple sequence alignment of the genomic region located between these two genes using sequence derived from our de novo DL238 and QX1211 assemblies, as well as available genomes from other Caenorhabditis species. QX1211 and ECA36 share the same haplotype in this region. Both DL238 and QX1211 carry a pseudogenized version of sup-35. pha-1 is missing from DL238 but pseudogenized in QX1211. In the four Caenorhabditis genomes available (C. briggsae, C. remanei, $C$. brenneri and C. angaria), the synteny of hmt-1, Y48A6C.4, and Y47D3A.29 was conserved. The synteny was also conserved in DL238 and QX1211, but altered in the N2 haplotype. Based on this alignment, the most likely scenario is that the sup-35/pha-1 element formed in the lineage leading to C. elegans. sup-35 and pha-1 were originally located next to each other in this ancestral haplotype and likely active. DL238, QX1211 and ECA36 carry a pseudogenized version of this ancestral haplotype. The ancestral haplotype also experienced an inversion affecting sup-35 and Y48A6C.4, placing Y48A6C.4 in between sup-35 and pha-1 and leading to the haplotype shared by the other 149 isolates tested, including N2. 


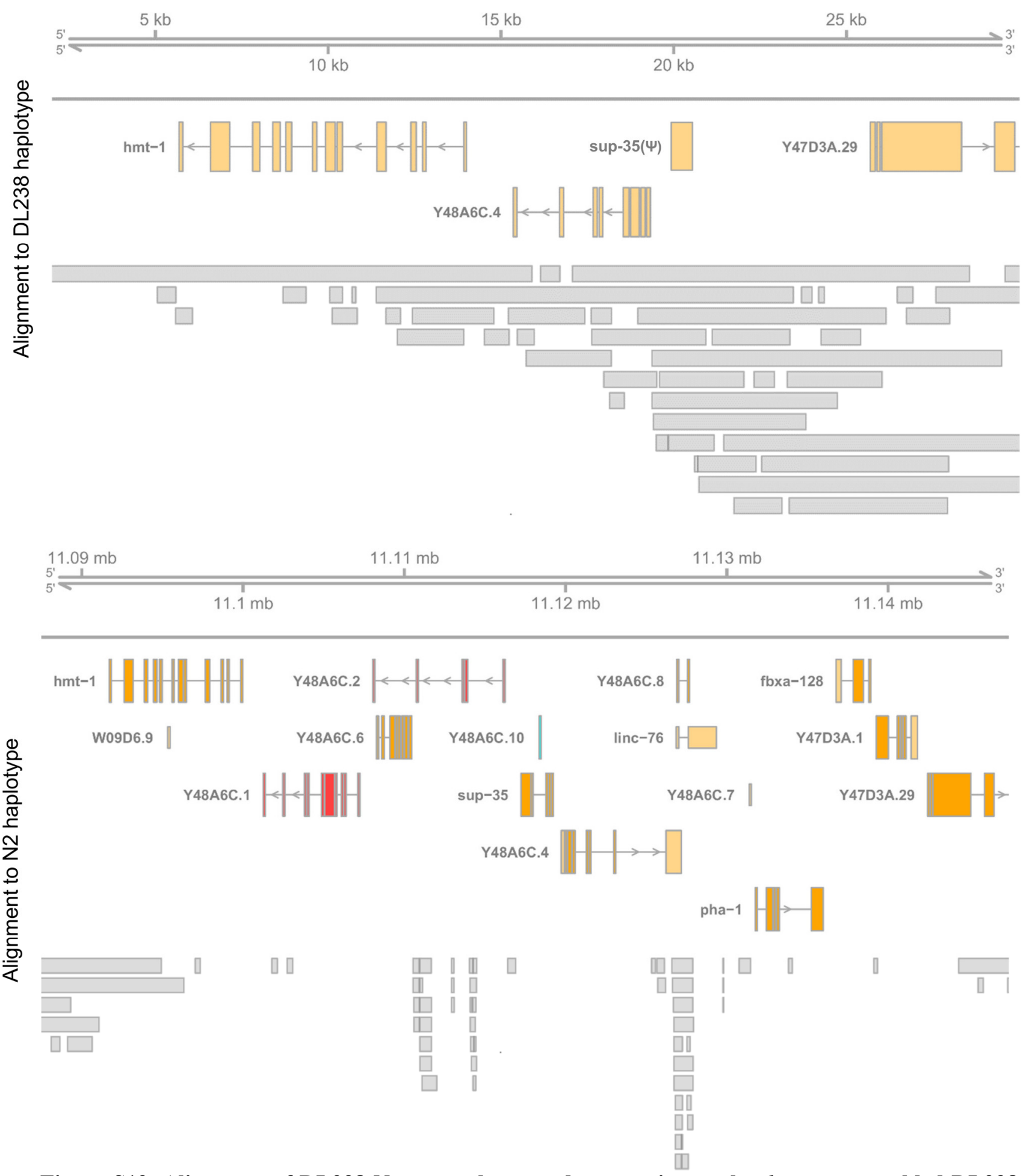

Figure S12. Alignment of DL238 Nanopore long read sequencing to the de novo assembled DL238 haplotype (top) and the N2 haplotype (bottom). Alignment to either the DL238 or the N2 haplotype is shown, along with the gene model in the region. sup-35(U) is the pseudogenized version of sup-35 found in DL238. The assembled DL238 haplotype is supported and independently validated by long reads that align across the region. 
bioRxiv preprint doi: https://doi.org/10.1101/112524; this version posted March 1, 2017. The copyright holder for this preprint (which was not certified by peer review) is the author/funder, who has granted bioRxiv a license to display the preprint in perpetuity. It is made available under aCC-BY-NC-ND 4.0 International license.

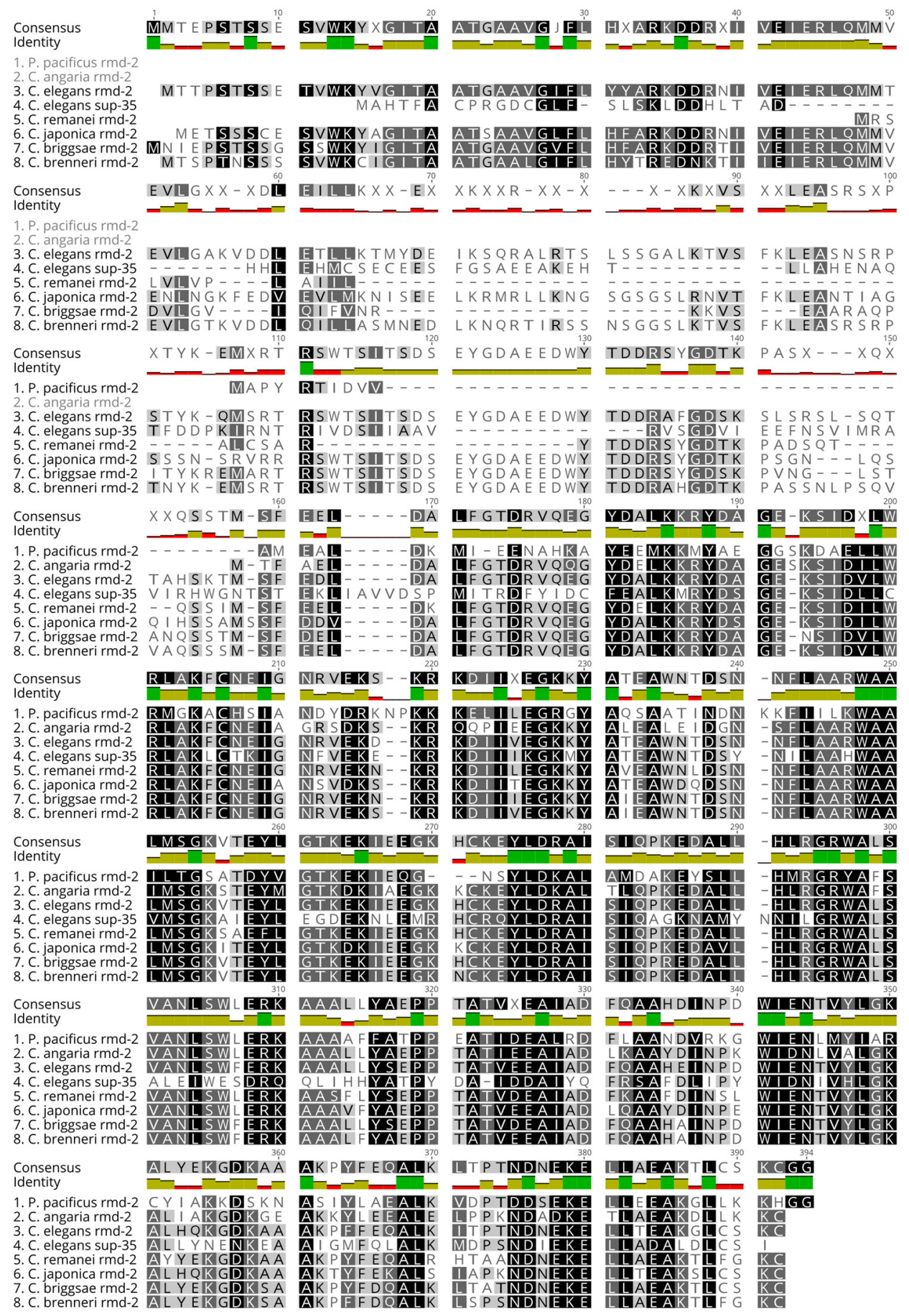

Figure S13. Protein alignment of sup-35 against $r m d-2$ from Caenorhabditis. Darker colors denote higher conservation. Similarly, conservation is also indicated by the color identity bar on top (red: low conservation, green: complete conservation. yellow: moderate conservation). For $C$. elegans, the largest transcript of $r m d-2$ (C27H6.4b) was used. 


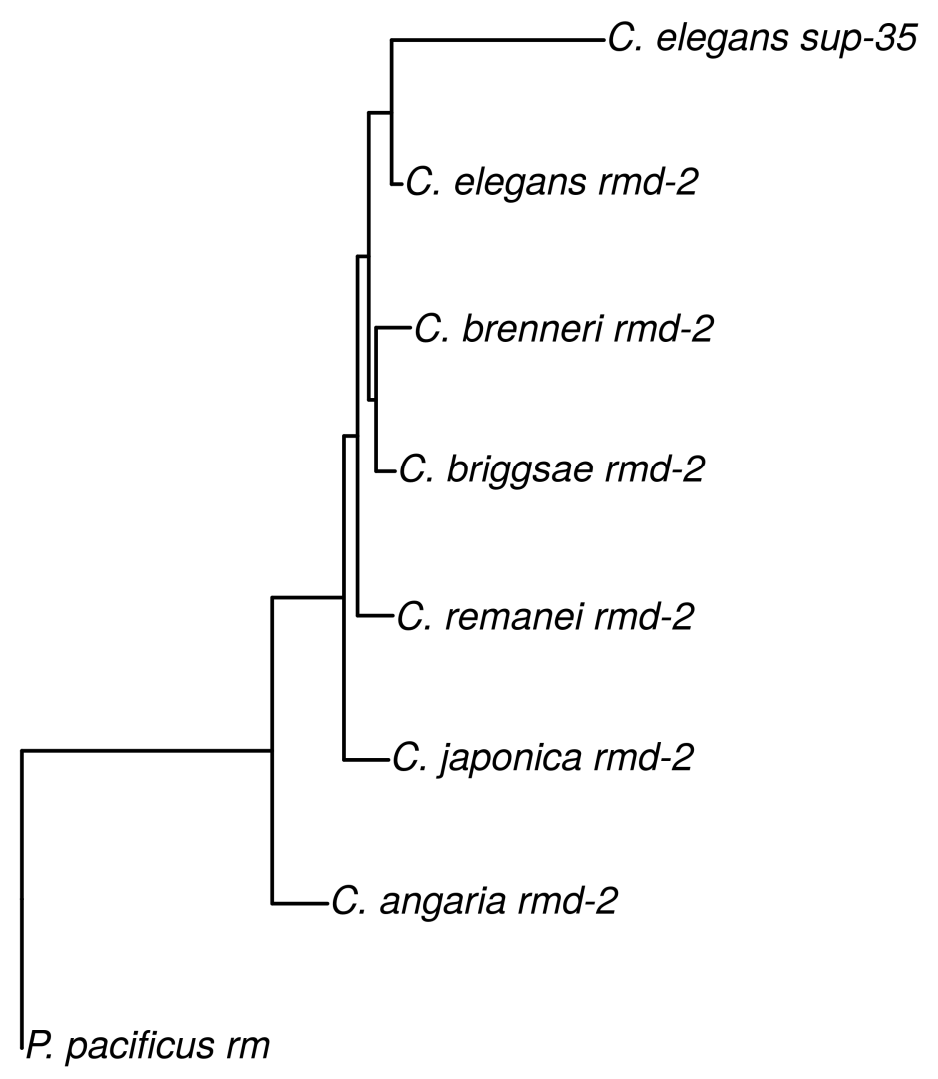

Figure S14. Phylogenetic tree of $r m d-2$ and sup-35 across diverse nematodes species. The phylogenetic tree for the cDNA of $r m d-2$ was constructed as described for Y48A6C.4. C. elegans $r m d-2$ is more closely related related to sup-35 than to orthologous $r m d-2$, suggesting that sup-35 arose from a duplication of $r m d$ 2 in the lineage leading to C. elegans. 


\section{Supplementary Tables}

\section{cơ $\left\|\quad q^{\prime}\right\| \times\left\|\sigma^{\prime \prime} \quad q^{\prime \prime}\right\| \times \| \sigma^{\prime \prime}$}

\begin{tabular}{|c|c|c|c|c|c|c|c|c|c|c|}
\hline Isolate & $\begin{array}{c}\text { peel-1/zeel-1 } \\
\text { Status }\end{array}$ & Seeded & Dead & Lethality & Seeded & Dead & Lethality & Seeded & Dead & Lethality \\
\hline BRC20067 & Inactive & 450 & 0 & $0 \%$ & 210 & 10 & $4.76 \%$ & 164 & 1 & $0.61 \%$ \\
\hline ED3012 & Active & 417 & 97 & $23.26 \%$ & 245 & 115 & $46.94 \%$ & 276 & 12 & $4.35 \%$ \\
\hline JT11398 & Active & 215 & 88 & $40.93 \%$ & 175 & 95 & $54.29 \%$ & 305 & 141 & $46.23 \%$ \\
\hline JU1400 & Active & 733 & 176 & $24.01 \%$ & 123 & 68 & $55.28 \%$ & 185 & 5 & $2.70 \%$ \\
\hline JU1652 & Active & 110 & 41 & $37.27 \%$ & 185 & 90 & $48.65 \%$ & 184 & 88 & $47.83 \%$ \\
\hline MY1 & Inactive & 420 & 113 & $26.90 \%$ & 248 & 7 & $2.82 \%$ & 125 & 57 & $45.60 \%$ \\
\hline MY16 & Active & 499 & 198 & $39.68 \%$ & 182 & 100 & $54.95 \%$ & 404 & 169 & $41.83 \%$ \\
\hline NIC195 & Active & 377 & 170 & $45.09 \%$ & 232 & 127 & $54.74 \%$ & 166 & 73 & $43.98 \%$ \\
\hline NIC199 & Active & 207 & 55 & $26.57 \%$ & 153 & 79 & $51.63 \%$ & 183 & 5 & $2.73 \%$ \\
\hline CX11271 & Inactive & 689 & 117 & $17.00 \%$ & 238 & 1 & $0.4 \%$ & 490 & 197 & $42.82 \%$ \\
\hline
\end{tabular}

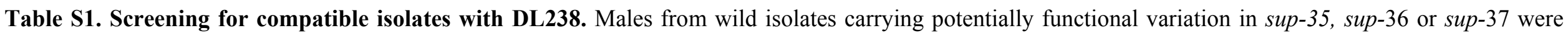

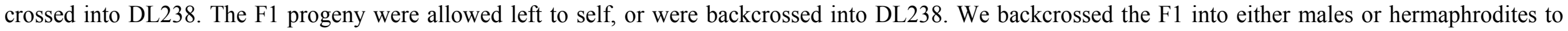

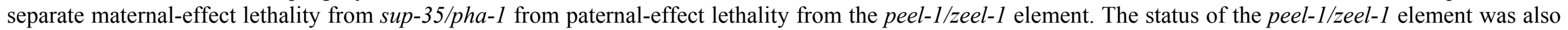
predicted by observing the sequencing in the isolates. 
bioRxiv preprint doi: https://doi.org/10.1101/112524; this version posted March 1, 2017. The copyright holder for this preprint (which was not certified by peer review) is the author/funder, who has granted bioRxiv a license to display the preprint in perpetuity. It is made available under aCC-BY-NC-ND 4.0 International license.

\begin{tabular}{|c|c|c|}
\hline Strain & Genotype & Comments \\
\hline $\mathrm{N} 2$ & Wild type reference & Isolated in Bristol, UK \\
\hline DL238 & Wild isolate & Isolated in Manuka National Reserve, Hawaii, USA \\
\hline CB4856 & Wild isolate & $\begin{array}{l}\text { Isolated in Honolulu, Hawaii, USA. It carries niDf9 I. } \\
\text { niDf9 designates the } 19 \mathrm{~kb} \text { deficiency spanning peel-1 } \\
\text { and zeel-1 }\end{array}$ \\
\hline QX1211 & Wild isolate & Isolated in California, USA. \\
\hline JK574 & fog-2(q71) $V$ & Hermaphrodites do not produce sperm and must outcross \\
\hline QX2323 & $q q \operatorname{Ir} 38[f o g-2(q 71), \mathrm{N} 2>\mathrm{DL} 238] \mathrm{V} *$ & $\begin{array}{l}\text { qqIr } 38 \text { is an introgression of the fog-2(q71) allele into } \\
\text { DL238 by performing eight rounds of backcross using } \\
\text { DL238 males. }(*) \text { This strain is also homozygous for the } \\
\text { sup-35/pha-1 N2 haplotype and was used to map the } \\
\text { incompatibility. }\end{array}$ \\
\hline QX2327 & $q q \operatorname{Ir} 39[f o g-2(q 71), \mathrm{N} 2>\mathrm{DL} 238] \mathrm{V}$ & $\begin{array}{l}\text { qqIr38 is an introgression of the fog-2(q71) allele into } \\
\text { DL238. QX2323 was further backcross two times using } \\
\text { DL238 hermaphrodites. This resulting strain is } \\
\text { homozygous for the sup-35/pha-1 DL238 haplotype. }\end{array}$ \\
\hline QX1198 & $q q \operatorname{Ir} 5[n i D f 9, \mathrm{CB} 4856>\mathrm{N} 2] \mathrm{I}$. & $\begin{array}{l}q q \operatorname{Ir} 5 \text { is a } 140-370 \mathrm{~kb} \text { introgression from CB4856 into } \\
\text { N2. This includes the inactive peel-1/zeel-1 haplotype of } \\
\text { CB } 4856 \text { (niDf9). }\end{array}$ \\
\hline GE346 & sup-35(e2223)pha-1(e2123) dpy-18(e499) III. & $\begin{array}{l}\text { sup-35(e2223) suppresses the temperature sensitive } \\
\text { embryonic lethality of pha-1(e2123), which is active at } \\
20 \mathrm{C} \text { and inactive at } 25 \mathrm{C} \text {. Dumpy. }\end{array}$ \\
\hline QX2329 & $\begin{array}{l}q q \operatorname{Ir} 5[n i D f 9, \mathrm{CB} 4856>\mathrm{N} 2] \mathrm{I} ; \text { sup-35(e2223) pha- } \\
1(e 2123) d p y-18(e 499) \text { III }\end{array}$ & $\begin{array}{l}\text { sup-35(e2223) in a N2 background with inactive peel- } \\
\text { 1/zeel-1 }\end{array}$ \\
\hline OH10050 & otIs317 [mgl-1::mCherry + pha-1(+)] & $\begin{array}{l}\text { pha- } 1 \text { overexpression transgene. Integrated in an } \\
\text { autosome, recues pha-1(e2123) }\end{array}$ \\
\hline QX2328 & $q q \operatorname{Ir} 40[$ otls317, N2 > DL238] & $\begin{array}{l}\text { pha- } 1 \text { overexpression transgene introgressed into DL } 238 \\
\text { by performing } 8 \text { round of backcross and selection. }\end{array}$ \\
\hline QX2330 & $\begin{array}{l}q q \operatorname{Ir} 5[\text { niDf9, CB4856 > N2] I; otIs } 317[\mathrm{mgl}- \\
1:: \mathrm{mCherry}+\text { pha- } 1(+)]\end{array}$ & $\begin{array}{l}\text { pha-1 overexpression transgene in an N2 background } \\
\text { with inactive peel-1/zeel-1 }\end{array}$ \\
\hline JT11398 & Wild isolate & Isolated in Seattle, WA, USA \\
\hline JU1652 & Wild isolate & Isolated in Montevideo, Uruguay \\
\hline JU1400 & Wild isolate & Isolated in Sevilla, Spain \\
\hline MY1 & Wild isolate & Isolated in Lingen, Germany \\
\hline CX11254 & Wild isolate & Isolated in Pasadena, CA, USA \\
\hline NIC199 & Wild isolate & Isolated in Ponta Delgada, Portugal \\
\hline BRC20067 & Wild isolate & Isolated in Taiwan \\
\hline ED3012 & Wild isolate & Isolated in Edinburgh, UK \\
\hline
\end{tabular}




\begin{tabular}{lll} 
MY16 & Wild isolate & Isolated in Münster, Germany \\
NIC195 & Wild isolate & Isolated in Ilha do Pico, Portugal \\
\hline
\end{tabular}

\section{Table S2: Strains used in the study}

